\title{
A new sawtooth control mechanism relying on toroidally propagating ion cyclotron resonance frequency waves: Theory and Joint European Torus tokamak experimental evidence ${ }^{a)}$
}

\author{
J. P. Graves, ${ }^{1, \text { b) }}$ I. T. Chapman, ${ }^{2}$ S. Coda, ${ }^{1}$ T. Johnson, ${ }^{3}$ M. Lennholm, ${ }^{4}$ and JET-EFDA \\ Contributors ${ }^{\mathrm{C})}$ \\ JET-EFDA, Culham Science Centre, Abingdon OX14 3DB, United Kingdom \\ ${ }^{1}$ École Polytechnique Fédérale de Lausanne (EPFL), Centre de Recherches en Physique des Plasmas, \\ Association EURATOM-Confédération Suisse, 1015 Lausanne, Switzerland \\ ${ }^{2}$ Euratom/CCFE Fusion Association, Culham Science Centre, Abingdon, United Kingdom \\ ${ }^{3}$ Euratom-VR Association, EES, KTH, SE-100 44 Stockholm, Sweden \\ ${ }^{4}$ EFDA-JET CSU, Culham Science Centre, Abingdon OX14 3DB, United Kingdom
}

(Received 20 November 2009; accepted 7 January 2010; published online 20 May 2010)

\begin{abstract}
The sawtooth control mechanism in plasmas employing toroidally propagating ion cyclotron resonance waves is extended. The asymmetrically distributed energetic passing ions are shown to modify the ideal internal kink mode when the position of the minority ion cyclotron resonance resides within a narrow region close to the $q=1$ surface. An analytical treatment of the internal kink mode in the presence of model distribution function with parallel velocity asymmetry is developed. The fast ion mechanism explains the strong sensitivity of sawteeth to resonance position, and moreover is consistent with dedicated Joint European Torus [F. Romanelli, Nucl. Fusion 49, 104006 (2009)] experiments which controlled sawteeth despite negligible current drive.
\end{abstract}

[doi:10.1063/1.3363201]

\section{INTRODUCTION}

The need for effective control of sawteeth has been well documented over the past few years. Due to the stabilizing role of trapped alpha particles, sawteeth are expected to be strongly stabilized in the international thermonuclear experimental reactor (ITER). ${ }^{1}$ The collapse radius and crash amplitude has been predicted to be so large in the ITER (Ref. 2) that coupling is likely to occur with instabilities located at other rational surfaces. Evidence of interaction between large sawteeth and neoclassical tearing modes (NTMs) has been observed $^{3,4}$ in the Joint European Torus (JET), ${ }^{5}$ while discharges with smaller regular sawteeth are found to have increased core confinement, and are less likely to be coupled to confinement degrading NTMs. Hence, it is seen that greater understanding and eventual control over the mechanisms that determine sawtooth stability is required. The key to this will be control over the interaction between fast minority ion dynamics and magnetohydrodynamic (MHD) stability.

While fast trapped ions are known to stabilize sawteeth, ${ }^{3,6,7}$ this paper demonstrates that under certain conditions ${ }^{8,9}$ energetic ions can also effectively destabilize sawteeth. Sawtooth control from energetic ions injected with near tangential unbalanced neutral beams has already been demonstrated analytically ${ }^{8}$ and numerically. ${ }^{10}$ It was found that an energy fluxlike quantity $\int d v^{3}\left(v^{2} / 2\right) v_{\|} \partial F / \partial r$ of the fast ion distribution $F$ at the $q=1$ surface has a markable effect on the internal kink stability. The energy flux clearly coincides with fast ion parallel currents, since both require

\footnotetext{
a) Paper GI3 2, Bull. Am. Phys. Soc. 54, 95 (2009).

${ }^{b)}$ Invited speaker.

${ }^{c}$ See the Appendix of F. Romanelli et al., Fusion Energy Conference 2008 (Proceedings of the 22nd International FEC Geneva, 2008), IAEA (2008).
}

parallel velocity asymmetries in the fast ion distribution function. The effect on the internal kink mode enters through finite orbit width corrections of the passing ions, which increases with the kinetic energy of the particles, and with pitch angles closer to the trapped-passing boundary. By extending the analysis and conclusions of Ref. 9, we show in this paper that JET plasmas with $-90^{\circ}$ phased off-axis ICRH share the same instability mechanism as the unbalanced neutral beam injection (NBI) scenarios mentioned above. The counter propagating waves yield asymmetric distributions of passing ions, which are, of course, consistent with the fast ion currents evaluated in such discharges. ${ }^{11}$ Dedicated SELFO (Ref. 12) RF wave-field and fast ion distribution simulations obtain, in particular, the parallel velocity asymmetry in the passing ion distribution function and their deposition. Analytical and full numerical calculations of the internal kink mode with the JET ICRH distribution functions demonstrate ideal instability when the deposition of $-90^{\circ}$ phased ions is very close to the $q=1$ radius. Such is the sensitivity to the location of deposition, and the magnitude of the effect, that this fast ion mechanism dominates over the previously assumed classical mechanism (e.g., Refs. 13, 14, and 11) relating to the change in the magnetic shear due the fast ions, and the resulting effect on resistive MHD and ideal MHD stability. Furthermore, the fast ion mechanism is independent of the bulk plasma drag current, which is expected ${ }^{15}$ to limit the net ion cyclotron current drive (ICCD) efficiency of the proposed ICRH system for ITER, and thus, diminish shear modification and the classical sawtooth control mechanism.

In order to address the issue that sawtooth control is due to the direct effect of fast ions, experiments have been devised where the current drive is small. In the experiments presented here, the net ion cyclotron driven current was sig- 
nificantly reduced by choosing ${ }^{3} \mathrm{He}$ minority ICRH, since the current dragged ${ }^{16,13}$ by the background plasma tends to cancel the ${ }^{3} \mathrm{He}$ current in the relevant region of the tokamak (provided that the effective charge of the plasma is close to that of ${ }^{3} \mathrm{He}$, where $Z_{\text {eff }}<2$ typically). It is shown that sawtooth control employing low concentration minority ${ }^{3} \mathrm{He}$ is nevertheless effective, thus strengthening the likelihood of the dominant effect of the fast ion mechanism, ${ }^{9}$ and moreover, demonstrating the viability of sawtooth control using ICRH in ITER, ${ }^{1}$ which is primarily and routinely expected to employ ${ }^{3} \mathrm{He}$ minority. These experiments, and earlier hydrogen minority pulses, ${ }^{17}$ are used as a platform to compare analytically derived internal kink stability with full numerical simulations.

The paper is organized as follows. In Sec. II, the internal kink mode calculations are derived, with particular attention given to the effects of asymmetrically distributed passing ions (the distribution function remains general except for an expansion in orbit width, which assumes that the energies of the fast ions are not too large). In Sec. III, the important features of the JET ICRH distribution functions are explored and modeled analytically. This information is used to evaluate the internal kink stability for JET demonstration discharges, and comparisons are made with full numerical internal kink solutions using SELFO (Ref. 12) and HAGIS. ${ }^{18}$ Here, HAGIS evaluates the fast particle contribution to the perturbed distribution function, and ultimately the growth rate, by following the guiding center motion of the population of ions in the presence of the internal kink perturbation. The analytical treatment contained in this manuscript enables identification of the fast ion growth rate in terms of the radial gradient of the fast ion current, thus assisting interpretation of existing experiments, and the design of experiments employed to test the theoretical mechanism. Finally, Sec. IV details minority ${ }^{3} \mathrm{He}$ experiments and the corresponding simulations which demonstrate the importance of the fast ion mechanism. Conclusions and discussions are reserved for the final section.

\section{STABILITY OF INTERNAL KINK MODE WITH ASYMMETRIC ION DISTRIBUTIONS}

We now set out to evaluate the stability of the internal kink mode in the presence of the fast ions. The motivation of this section is to identify the effects of finite orbit widths and parallel velocity asymmetry for an arbitrary distribution function. Consequently, the results of this section will generalize the fast ion contributions identified in Ref. 8, which was valid for a slowing down distribution with parallel velocity asymmetry and a delta function in pitch angle (such that all particles are deeply passing $v_{\perp}=0$ ), with application essentially only to unbalanced strongly tangential neutral beams. The relevant ordering employed is $\beta_{h} \sim \beta_{c} \sim O\left(\epsilon^{2}\right)$, where $\beta_{h, c}=2 \mu_{0} P_{h, c} / B_{0}^{2}$ is the ratio of hot, or core, particle pressure and the magnetic pressure, and $\epsilon$ is the inverse aspect ratio. Quantities attributed to the fast ion population, are for convenience, henceforth absent of a subscript " $h$." The response of energetic ions to the internal kink mode is obtained by perturbing the hot ion distribution $F\left(\mathcal{E}, \mathcal{P}_{\phi}, \mu, \sigma\right)$ with respect to the constants of motion. ${ }^{19}$ We note that for discharges employing ICRH auxiliary heating only, the equilibrium electric field $E=-\nabla \Phi$ provides only a small correction to the actual conserved total energy $\mathcal{E}+Z e \Phi / m$, and so the equilibrium electric field, and its corresponding plasma rotation, is neglected here. Focusing on ideal MHD modes in a low $\beta$ and small $\epsilon$ tokamak, we write down a solution ${ }^{19,20}$ to the perturbed distribution function $\delta F=\delta F_{f}+\delta F_{k}$ which treats wide radial drift excursion. From Refs. 19 and 20

$$
\delta F_{f}=-(Z e / m)\left(\boldsymbol{\xi} \cdot \nabla \psi_{p}\right) \frac{\partial F}{\partial \mathcal{P}_{\phi}}
$$

is the adiabatic (fluid) contribution, $\boldsymbol{\xi}=\hat{\boldsymbol{\xi}} \exp (-i n \phi-i \omega t)$ is the MHD displacement with $\hat{\boldsymbol{\xi}}=\Sigma_{m} \hat{\boldsymbol{\xi}}_{m} \exp (-\operatorname{im} \theta)$, and the nonadiabatic (kinetic) contribution $\delta F_{k}$ can be approximately written as "bounce time" $\tau_{b}=2 \pi / \omega_{b}$ periodic function of time:

$$
\begin{aligned}
\delta F_{k}= & \sum_{l=-\infty}^{\infty} \delta F_{k}^{(l)} \exp \left[-i\left(\omega+l \omega_{b}+n\langle\dot{\phi}\rangle\right) t\right], \\
\delta F_{k}^{(l)}= & -\frac{\omega-n \omega_{*}}{\omega+n\langle\dot{\boldsymbol{\phi}}\rangle+l \omega_{b}} \frac{\partial F}{\partial \mathcal{E}} \\
& \times\left\langle\left(v_{\|}^{2}+\frac{v_{\perp}^{2}}{2}\right) \boldsymbol{\kappa} \cdot \boldsymbol{\xi}_{\perp} \exp \left[i\left(\omega+l \omega_{b}+n\langle\dot{\boldsymbol{\phi}}\rangle\right) t\right]\right\rangle,
\end{aligned}
$$

where $\omega_{*}=\partial F / \partial \mathcal{P}_{\phi}(\partial F / \partial \mathcal{E})^{-1}$ is the diamagnetic frequency, $\boldsymbol{\kappa}$ is the magnetic curvature vector, and $\langle X\rangle=\left(1 / \tau_{b}\right) \int_{0}^{\tau_{b}} d t X$.

In the limit of zero radial drift excursion, or orbit width $\Delta_{r}$, the adiabatic (or fluid) contribution is simply a result of convective " $c$ " motion perpendicular to the field line, $\delta F_{c}=-\boldsymbol{\xi}_{\perp} \cdot \boldsymbol{\nabla} F$. Meanwhile, $\delta F_{k}$ is associated with the parallel dynamics, and is often referred to as the response due to kinetic-compressibility In particular, replacing the drift kinetic treatment employed here with the ideal MHD model, one would substitute $\delta F_{k}$ defined above with $\delta F_{k n c}$ $=-\gamma F \boldsymbol{\nabla} \cdot \boldsymbol{\xi}$, the nonconvective " $n c$ " stabilizing effect of compressibility. Assuming an isotropic equilibrium distribution function, taking a second velocity moment of $\delta F_{c}+\delta F_{k n c}$ simply yields the MHD perturbed pressure $\delta P_{c}=-\boldsymbol{\xi}_{\perp} \cdot \nabla P$ $-\gamma P \boldsymbol{\nabla} \cdot \boldsymbol{\xi}$. The MHD model provides a very poor description of the parallel dynamics of energetic particles, and clearly cannot describe resonant wave-particle interaction. However, MHD does a better job of representing perpendicular dynamics. In particular, the perpendicular component of the MHD equation of motion, in which $\delta P_{c}=-\boldsymbol{\xi}_{\perp} \cdot \nabla P$ (the second moment of $\delta F_{c}$ ) appears explicitly, describes the essential perpendicular dynamics of a hybrid kinetic-MHD treatment. Nevertheless, in additional to the well known nonconvective effects associated with the kinetic response $\delta F_{k}$, finite orbit widths introduce additional nonconvective effects in $\delta F_{f}{ }^{8}$, As we will see, by expanding $\delta F_{f}$ with respect to small orbit width, we are able to identify the nonconvective correction associated with the fluid contribution: ${ }^{8,9}$ 


$$
\delta F_{f n c}=-(Z e / m)\left(\boldsymbol{\xi} \cdot \nabla \psi_{p}\right) \frac{\partial F}{\partial \mathcal{P}_{\phi}}+\boldsymbol{\xi}_{\perp} \cdot \boldsymbol{\nabla} F
$$

It is in this correction that the effect of parallel velocity asymmetry on the internal kink mode arises, and the on resulting observable affect on sawteeth.

In order to relate Eqs. (1) and (2) to a potential energy $\delta W$, we recall that $\delta W$ is defined in terms of the perturbed force $\delta^{\dddot{r}}$ via $\delta W=-(1 / 2) \int d^{3} x \boldsymbol{\xi}_{\perp}^{*} \cdot \boldsymbol{\delta}^{\sharp}$, where

$$
\boldsymbol{\delta}^{f}=\boldsymbol{\delta} \boldsymbol{j} \times \boldsymbol{B}+\boldsymbol{j} \times \boldsymbol{\delta B}-\boldsymbol{\nabla} \cdot \underline{\underline{\delta P}} .
$$

Now, the fast ions primarily influence the linear perturbed force $\boldsymbol{\delta} f$ through the perturbed pressure tensor $\underline{\underline{\delta P}}$. This is despite the fact that the fast ion distribution function also yields a significant equilibrium current. As discussed in Ref. 20, the flux averaged fast ion current contributes an order $\epsilon$ correction to the safety factor, and this in turn plays a role in the MHD field line bending contribution to the internal kink mode. The latter is, however, minimized to insignificant ordering by the top-hat leading order displacement $\hat{\boldsymbol{\xi}}_{1} \cdot \nabla r=\boldsymbol{\xi}_{r} H\left[r_{1}-r\right]$, where $H$ is the Heaviside step function, and $r_{1}$ is the radius at which $q=1$. Moreover, even though the poloidal modulation of the fast ion current is of order unity, it does not contribute to $\delta W$ at significant order. The effects of fast ions are found to enter the dynamics entirely through the perturbed pressure tensor. The fast ion pressure tensor $\underline{\underline{\delta P}}$ is of course obtained by evaluating the second velocity moments of $\delta F$, and results in

$$
\delta W=\frac{1}{2} m \int d^{3} x \int d^{3} v \boldsymbol{\kappa} \cdot \boldsymbol{\xi}_{\perp}^{*}\left(v_{\|}^{2}+\frac{v_{\perp}^{2}}{2}\right) \delta F_{h},
$$

where

$$
\begin{aligned}
& \int_{\text {all v }} d v^{3}=\sum_{\sigma} \pi \int_{0}^{\infty} d \mathcal{E}(2 \mathcal{E}) \int_{0}^{1 / B} d \lambda \frac{B}{\left|v_{\|}\right|} \text {and } \\
& \int d x^{3}=2 \pi \int_{0}^{2 \pi} d \theta \int_{0}^{a} d r R r .
\end{aligned}
$$

The kinetic contributions to $\delta W$ are examined first. Due to the high energy of the ICRH species, such that $\omega_{* h}, \omega_{b}$, and $\langle\dot{\phi}\rangle$ are much larger than the frequency of the $n=1$ mode responsible for sawteeth, it is appropriate to employ the approximation $\omega=0$ in Eq. (3). For both trapped ions and passing ions, the largest kinetic contribution to low frequency sawtooth modes occurs in the absence of bounce harmonics, i.e., for $l=0$. The small contribution (when $\omega=0$ ) that exists when one takes $l=1$ for the passing ion response has been examined previously, ${ }^{8,21,22}$ and is found to be small, and is thus neglected here. To make progress, we need to expand $F$ in orders of the orbit width $\Delta_{r}$, so that $F=F_{0}+F_{1}+\ldots$. We note that another constant of motion, defined by $\mathcal{P}_{\phi}$, is the temporal average of the particles' minor radius, $\bar{r}$, over a full toroidal transit, i.e., $\bar{r}=\tau_{b}^{-1} \int_{0}^{\tau_{b}} d t r(t)$, where $\tau_{b}$ is the bounce time, or transit time, for, respectively, trapped or passing particles. Writing $r(t)=\bar{r}+\Delta_{r}(t)$ we have

$$
F_{0}=\left.F(\mathcal{E}, \mu, \bar{r})\right|_{\vec{r} \rightarrow r} \text { and } F_{1}(\mathcal{E}, \mu, r)=-\Delta_{r} G_{0}(\mathcal{E}, \mu, r),
$$

and

$$
G_{0}(\mathcal{E}, \mu, r)=\left.G(\mathcal{E}, \mu, \bar{r})\right|_{\bar{r} \rightarrow r} \text { and } G=\frac{\partial F}{\partial \bar{r}},
$$

with

$$
\Delta_{r}=\frac{q}{r \Omega_{c}}\left(\left|v_{\|}\right| R-p R_{0}^{2} q \omega_{b}\right),
$$

where $\sigma$ is the sign of $v_{\|}, p=1$ for passing particles, $p=0$ for trapped particles, $\omega_{b}=2 \pi / \tau_{b}$, and $\Omega_{c}=e Z B_{0} / m$. Defining $F^{ \pm}=F(\sigma)$ and $G^{ \pm}=G(\sigma)$, etc., to separate the contributions of particles with $v_{\|}>0$ and $v_{\|}<0$, we now have for the passing " $p$ " and trapped " $t$ " ions the leading order (in orbit width) kinetic (" $k$ ") response (by setting $\omega=0$ and $l=0$ in $\left.\delta F_{k}\right)$ :

$$
\begin{aligned}
\delta \hat{W}_{k p}= & -\left(\frac{2}{\pi}\right)^{1 / 2} \frac{2}{\epsilon_{1}^{2} R_{0}} \int_{0}^{r_{1}} d r \int_{0}^{1} d y^{2}\left[\frac{2}{\pi}\left(G_{3}+\frac{G_{4}}{2}\right)\right]^{2} \\
& \times\left(\frac{q}{\Omega_{c}}\right)\left(\frac{e T_{\perp}}{m}\right)^{1 / 2}\left(\frac{2 \mu_{0}}{B_{0}^{2}}\right)\left(C^{+}-C^{-}\right)
\end{aligned}
$$

and

$$
\begin{aligned}
\delta \hat{W}_{k t}= & -\frac{3}{2^{3 / 2} \pi \epsilon_{1}^{1 / 2}} \int_{0}^{r_{1}} d r\left(\frac{r}{r_{1}}\right)^{3 / 2} \int_{0}^{1} d k^{2} \frac{F_{q}^{2}}{K F_{d}}\left(\frac{2 \mu_{0}}{B_{0}^{2}}\right) \\
& \times\left(D_{t}^{+}+D_{t}^{-}\right)
\end{aligned}
$$

where $\delta \hat{W}=\delta W /\left(2 \pi^{2} R_{0} \xi_{r}^{2} \epsilon_{1}^{2} B_{0}^{2} / \mu_{0}\right)$, the pitch angle $k^{2}$ $=\left[1-\lambda B_{0}(1-\epsilon)\right] / 2 \lambda B_{0} \epsilon$ for trapped ions and $y^{2}=1 / k^{2}$ for passing ions. Note that the upper limit $r_{1}$ above, and in the equations that follow, is a result of imposing the leading order eigenfunction which vanishes outside $r_{1}$. Furthermore, we have

$$
C^{\sigma}=\left(\frac{e T_{\perp}}{m}\right)^{-1 / 2} \frac{(m / 2)(\pi / 2)^{3 / 2}}{\left[y^{2}+\epsilon\left(2-y^{2}\right)\right]^{3}} \int_{0}^{\infty} d \mathcal{E}(2 \mathcal{E})^{2} G_{0}^{\sigma},
$$

together with

$$
D_{t}^{\sigma}=\frac{m(2 \pi / 3)}{\left[1+\epsilon\left(2 k^{2}-1\right)\right]^{5 / 2}} \int_{0}^{\infty} d \mathcal{E}(2 \mathcal{E})^{3 / 2} G_{0}^{\sigma}
$$

and the following passing ion pressure gradient related quantity will be required later

$$
D_{p}^{\sigma}=\frac{m(2 \pi / 3)}{\left[y^{2}+\epsilon\left(2-y^{2}\right)\right]^{5 / 2}} \int_{0}^{\infty} d \mathcal{E}(2 \mathcal{E})^{3 / 2} G_{0}^{\sigma} .
$$

In the above, we have used $\langle\dot{\phi}\rangle=q \omega_{b}$ for passing ions (valid at the required ordering in Larmor radius and orbit width), and $\langle\dot{\phi}\rangle=-q\left[\mathcal{E} F_{d}\left(r, k^{2}\right) /\left(r R_{0} \Omega_{c}\right)\right]$ for trapped ions, where

$$
\begin{aligned}
F_{d}^{t}\left(r, k^{2}\right)= & \frac{2}{1+\epsilon\left(2 k^{2}-1\right)} \\
& \times\left[H_{1}\left(k^{2}\right)+2 s(r) H_{2}\left(k^{2}\right)-\Delta^{\prime \prime}(r) H_{3}\left(k^{2}\right)-\frac{\alpha}{4 q^{2}}\right],
\end{aligned}
$$

$s=(r / q) q^{\prime}$ is the magnetic shear, $\alpha=-2 R_{0} q^{2} P^{\prime} / B_{0}^{2}$ is the 
ballooning parameter $\Delta^{\prime \prime} \approx \alpha$ (where $\Delta$ is the Shafranov shift), while $H_{1}, H_{2}$, and $H_{3}$ are given by Eq. (10) of Ref. 23 (written there as $G_{1,2,3}$ ), and are entirely in terms of elliptic integrals. Moreover, the definition $F_{q}, G_{3}$, and $G_{4}$, as well as other expressions are given in the appendix. We note however that $F_{q}, G_{3}$, and $G_{4}$ involve integration of $\cos (\phi$ $-\langle\dot{\phi}\rangle t)$ over the full poloidal circuit. In order to make progress we use $\phi-\langle\dot{\phi}\rangle t=q \theta$ for the trapped particles, while for passing particles, we are at liberty to use the result $\phi-\langle\dot{\phi}\rangle t=q\left(\theta-\pi K\left[\theta / 2, y^{2}\right] / K\left[y^{2}\right]\right)$, where $K\left[\phi, y^{2}\right]$ is an incomplete elliptic integral of the first kind, and $K\left[y^{2}\right]=K\left[\pi / 2, y^{2}\right]$ is a complete elliptic integral of the first kind.

We note that $\delta \hat{W}_{k t}$ involves $G_{0}^{+}+G_{0}^{-}$, and thus contributes when the distribution is symmetric or not. Indeed, it is generally thought that $\delta \hat{W}_{k t}$ is responsible for conventional sawtooth stabilization by energetic ions. The leading order passing ion kinetic contribution, $\delta \hat{W}_{k p}$, scales as a finite orbit correction, ${ }^{8}$ since $\delta \hat{W}_{k p} / \delta \hat{W}_{k t}$ is proportional to $\left(q / \Omega_{c}\right)\left(e T_{\perp} / m\right)^{1 / 2} \sim \Delta_{r} / r$. Furthermore, it is important to note that $\delta \hat{W}_{k p}$ is proportional to $G_{0}^{+}-G_{0}^{-}$, and is therefore only nonzero when the distribution is asymmetric at the lowest order. We note that Eq. (10) generalizes the passing ion, finite orbit kinetic response of Eq. (14) in Ref. 24, now accounting for arbitrary distribution function. Nevertheless, as is the case for a simplified passing ion distribution, ${ }^{8} \delta \hat{W}_{k p}$ is seen once again to be cancelled out by part of the adiabatic response when finite orbit corrections are included.

We now go about solving for the adiabatic contribution, corresponding to Eq. (1). In order to separate zeroth order effects from finite orbit effects, we expand Eq. (1) about the flux label $r$. For this purpose we note that $\boldsymbol{\xi} \cdot \boldsymbol{\nabla} \psi$ $=r B_{0} \xi_{r} / q(r)$ and $\partial / \partial \mathcal{P}_{\phi}=\Omega_{c}^{-1}(q(\bar{r}) / \bar{r}) \partial / \partial \bar{r}, r / \bar{r}=1+\left(\Delta_{r} / r\right)$, $q(\vec{r}) / q(r)=1-\left(\partial \Delta_{r} / \partial r\right)$, where $\partial \Delta_{r} / \partial r=\Delta_{r} s(r) / r$. This then yields

$$
\delta F_{f}=\delta F_{f c}+\delta F_{f n c}
$$

with the convective contribution:

$$
\delta F_{f c}=-\xi_{r} G_{0},
$$

and the nonconvective contribution:

$$
\begin{aligned}
\delta F_{f n c}= & -\xi_{r} \frac{\Delta_{r}}{r}\left[(2-s) G_{0}-\left.\frac{y^{2}}{2}\left(2-y^{2}\right) \frac{\partial G_{0}}{\partial y^{2}}\right|_{r}\right. \\
& \left.-\left.\frac{\partial\left(r G_{0}\right)}{\partial r}\right|_{y^{2}}\right],
\end{aligned}
$$

where it is important to highlight that $\partial G /\left.\partial r\right|_{\lambda}=\partial G /\left.\partial r\right|_{y^{2}}$ $-\left(2-y^{2}\right)\left(y^{2} / 2\right) \partial G /\left.\partial y\right|_{r}$, must be taken into account when dealing with partial derivatives. We are reminded however, that $G_{0}$, defined in Eq. (7), is the radial derivative of $F$, with $\lambda$ kept constant, since $G=\partial F /\left.\partial \bar{r}\right|_{\lambda}$.

We now evaluate the $\delta W$ expressions corresponding to each of the terms in Eq. (11). Clearly Eq. (12) is simply the convective contribution $\delta F_{c}=-\boldsymbol{\xi}_{\perp} \cdot \nabla F$ described earlier, and leads to the usual adiabatic response in the absence of finite orbit effects:

$$
\begin{aligned}
\delta \hat{W}_{f t 0}= & \frac{3}{2^{3 / 2} \pi \epsilon_{1}^{1 / 2}} \int_{0}^{r_{1}} d r\left(\frac{r}{r_{1}}\right)^{3 / 2} \int_{0}^{1} d k^{2}\left(2 G_{1 t}+G_{2 t}\right) \\
& \times\left(\frac{2 \mu_{0}}{B_{0}^{2}}\right)\left(D_{t}^{+}+D_{t}^{-}\right), \\
\delta \hat{W}_{f p 0}= & \frac{3}{2^{3 / 2} \pi \epsilon_{1}^{1 / 2}} \int_{0}^{r_{1}} d r\left(\frac{r}{r_{1}}\right)^{3 / 2} \int_{0}^{1} d y^{2}\left(2 G_{1 p}+G_{2 p}\right) \\
& \times\left(\frac{2 \mu_{0}}{B_{0}^{2}}\right)\left(D_{p}^{+}+D_{p}^{-}\right) .
\end{aligned}
$$

The sum of Eqs. (14) and (15) is the lowest order (in orbit width) adiabatic energetic ion response, and is generally the same order of magnitude as the well known trapped ion kinetic response $\delta \hat{W}_{k t}$ of Eq. (10). In the isotropic limit $A=T_{\perp} / T_{\|}=1$, we find $\delta \hat{W}_{f t 0}+\delta \hat{W}_{f p 0}=0$. Meanwhile, the total fluid contribution comprises also the finite orbit response $\delta \hat{W}_{f p 1}$, so that $\delta \hat{W}_{f}=\delta \hat{W}_{f t 0}+\delta \hat{W}_{f p 0}+\delta \hat{W}_{f p 1}$. The nonconvective contribution $\delta F_{f n c}$ given by Eq. (13) is proportional to $\Delta r$, i.e., corresponding to Eq. (4) for the internal kink mode, and yields the following expression, written here in a convenient form upon integration by parts:

$$
\delta \hat{W}_{f p 1}=\delta \hat{W}_{r_{1}}+\delta \hat{W}_{y^{2}=1}+\delta \hat{W}_{f p 1}(\text { extra }),
$$

where each of the above terms are defined in the following. First of all,

$$
\begin{aligned}
\delta \hat{W}_{f p t}(\text { extra })= & \left(\frac{2}{\pi}\right)^{1 / 2} \frac{2}{\epsilon_{1}^{2} R_{0}} \int_{0}^{r_{1}} d r \int_{0}^{1} d y^{2} \\
& \times\left\{2 \left[\frac{\epsilon y^{2}}{2}-\left(\frac{y^{2}}{2}+\frac{\epsilon}{2} \frac{\partial}{\partial \epsilon}+\frac{y^{2}}{4}\left(2-y^{2}\right) \frac{\partial}{\partial y^{2}}\right)\right.\right. \\
& \left.\left.\times\left(\frac{2 G_{1}+G_{2}}{K\left[y^{2}\right]}\right)\right]\right\}\left(\frac{q}{\Omega_{c}}\right)\left(\frac{e T_{\perp}}{m}\right)^{1 / 2}\left(\frac{2 \mu_{0}}{B_{0}^{2}}\right) \\
& \times\left(C^{+}-C^{-}\right)
\end{aligned}
$$

is almost cancelled by the kinetic contribution for passing ions given by Eq. (9). It turns out that sum of the intregrands (in the $y^{2}$ integrals) of Eqs. (9) and (17) cancel for arbitrary $\epsilon$ except near the passing-trapped boundary $(y \approx 1)$, and due to the magnitude of $\delta \hat{W}_{r_{1}}$, it is legitimate to take $\delta \hat{W}_{f p 1}$ (extra) $+\delta \hat{W}_{k p}=0$. Thus, Eq. (10), which for arbitrary distribution function generalizes the passing ion finite orbit kinetic response of Eq. (14) in Ref. 24, is cancelled. The term has been shown ${ }^{8}$ to exactly cancel for the strongly parallel distribution employed in Refs. 24 and 8. The remaining terms in $\delta \hat{W}_{f p 1}$ are boundary terms. In particular we have the term evaluated on the passing side of the passing-trapped boundary $\left(y^{2}=1\right)$ :

$$
\begin{aligned}
\delta \hat{W}_{y^{2}=1}= & -\left(\frac{2}{\pi}\right)^{1 / 2} \frac{2}{\epsilon_{1}^{2} R_{0}} \int_{0}^{r_{1}} d r\left(\frac{\epsilon}{4}-\frac{2 G_{1}+G_{2}}{2 K\left[y^{2}\right]}\right)\left(\frac{q}{\Omega_{c}}\right) \\
& \times\left.\left(\frac{e T_{\perp}}{m}\right)^{1 / 2}\left(\frac{2 \mu_{0}}{B_{0}^{2}}\right)\left(C^{+}-C^{-}\right)\right|_{y^{2}=1} .
\end{aligned}
$$

We recall that the reflection of trapped particles requires that 

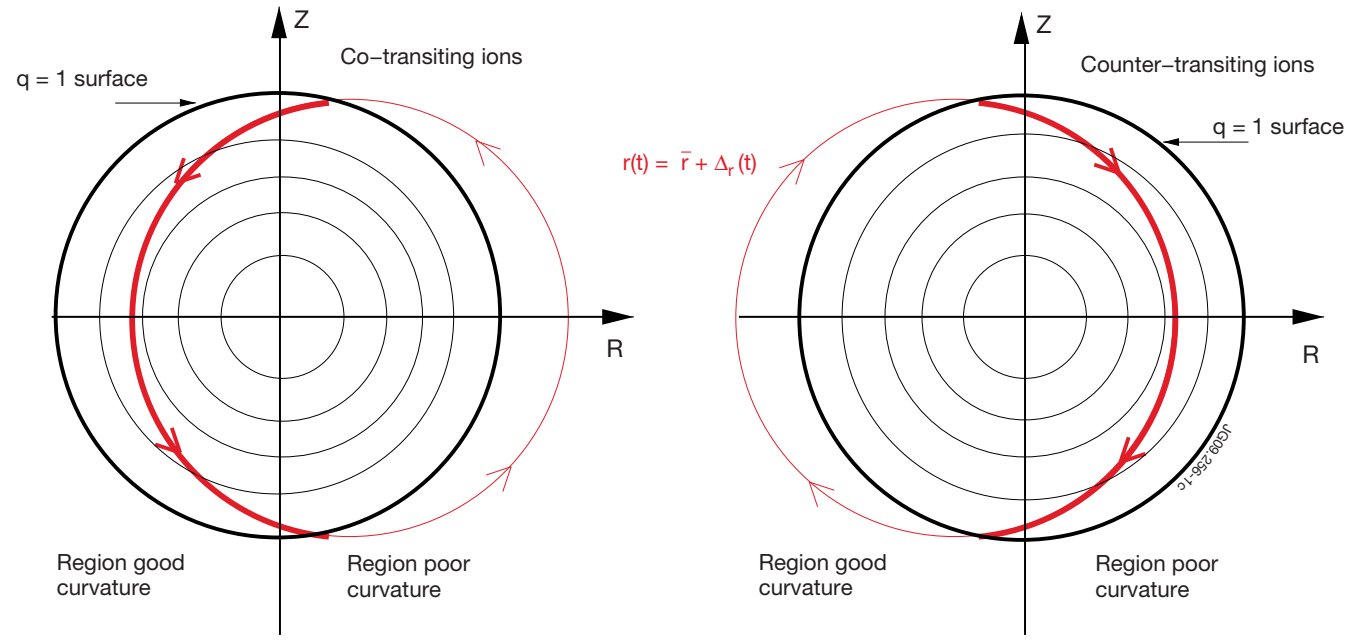

FIG. 1. (Color online) Plotting copassing and counter passing ions intersecting the $q=1$ surface.

$G_{0}^{+}-G_{0}^{-}=0$ in the trapped region, of velocity space, and so, if the distribution function is continuous across the passingtrapped boundary, we require that $C^{+}\left(y^{2}=1\right)-C^{-}\left(y^{2}=1\right)=0$, and hence $\delta \hat{W}_{y^{2}=1}=0$. Indeed, as discussed and implemented in, e.g., Refs. 25 and 26, slowing down and pitch angle scattering of fast ions due to collisions ensure that the distribution function is continuous across the passing-trappedtrapped boundary. Moreover, since the orbit width $\Delta_{r}$ is also continuous across the boundary, it therefore follows that the leading order (in orbit width) distribution function $F_{0}$ is also continuous with respect to pitch angle.

The only significant remaining finite orbit correction term is the following, evaluated at $r=r_{1}$ :

$$
\begin{aligned}
\delta \hat{W}_{r_{1}}= & -\left(\frac{2}{\pi}\right)^{1 / 2} \frac{2}{\epsilon_{1}} \int_{0}^{1} d y^{2}\left(\frac{\epsilon_{1} y^{2}}{2}-\frac{2 G_{1}+G_{2}}{K\left[y^{2}\right]}\right)\left(\frac{q}{\Omega_{c}}\right) \\
& \times\left.\left(\frac{e T_{\perp}}{m}\right)^{1 / 2}\left(\frac{2 \mu_{0}}{B_{0}^{2}}\right)\left(C^{+}-C^{-}\right)\right|_{r=r_{1}} .
\end{aligned}
$$

Equation (19) is valid for an arbitrary distribution function, and thus generalizes Eq. (5) of Ref. 8, which is valid for an asymmetric slowing down distribution with strongly parallel pitch angle. Note that, in order to compare with Ref. 8, we must take $g(l)=0$ in Eq. (5) of Ref. 8, which is valid for highly energetic particles in a plasma with finite magnetic shear, for which $\omega \ll k_{\|} v_{\|}{ }^{27,21}$ Here $g(l)$ arises from the $l=1$ bounce harmonic of the kinetic response $\delta F_{k}$, which has already been discussed and dismissed.

It is clear that $\delta W_{r_{1}}$ given by Eq. (19) above does not vanish if $G_{0}^{+}-G_{0}^{-} \neq 0$, since $\delta \hat{W}_{r_{1}}$ represents the effect of having nonzero parallel energy flux at the $q=1$ surface. The particular energy fluxlike quantity that matters is proportional to $\int d v^{3} G_{0}\left(V_{\perp}+v_{\|}^{2} / 2\right) v_{\|}$, and clearly this third moment of $\partial F_{0} / \partial r$ will be nonzero when there are localized fast ion currents. Clearly, $\delta \hat{W}_{r_{1}}$ is enhanced for increased finite orbit widths, which are in turn enhanced by loading the distribution function close to the passing-trapped boundary, and by increasing the thermal energies of the particles. The sensitivity of $\delta \hat{W}_{r_{1}}$ to the asymmetry in the lowest order distribution function means that stability is intrinsically linked to the sign and magnitude of the derivative of the lowest order fast ion current. These concepts are assisted by consideration of Fig. 1. Only ions which intersect $q=1$ contribute to Eq. (19). If an orbit is entirely always outside $r_{1}$, the particle does not contribute to the internal kink mode, due to the mode amplitude being zero outside $r_{1}$. If an orbit is constrained to be entirely inside $r_{1}$, then the integral of $\xi_{0} H[r(t)] \cos \theta(t)\left[v_{\perp}(t)^{2} / 2\right.$ $\left.+v_{\|}(t)^{2}\right] \Delta_{r}(t) / v_{\|}(t)$ along the path $t$ will not integrate to zero, but the similar adiabatic and nonadiabatic contributions sum to zero, as described above [Eqs. (9) and (17) sum approximately to zero, as originally discovered in Ref. 8]. For particles that do intersect, then the portion of $\xi_{0} H[r(t)] \cos \theta(t)$ $\times\left[v_{\perp}(t)^{2} / 2+v_{\|}(t)^{2}\right] \Delta_{r}(t) / v_{\|}(t)$ along the path $t$ that is inside $q=1$ contributes to the integral, and this region of the orbit is shown in bold in Fig. 1. If there are an equal number of coand counterpassing particles, the effect of particles intersecting $q=1$ is nullified. However, destabilization can occur when $\partial F\left(v_{\|}>0\right) / \partial r>\partial F\left(v_{\|}<0\right) / \partial r$, i.e., $G_{0}^{+}>G_{0}^{-}$. These identities occur, for example, with populations of ions created by unbalanced neutral beam injection. Destabilization will occur for off-axis $(\partial F / \partial r>0)$ with injection orientated along the plasma current $\left(F_{0}^{+}>F_{0}^{-}\right)$, or with on-axis beams $(\partial F / \partial r<0)$ with injection orientated counter to the plasma current $\left(F_{0}^{+}<F_{0}^{-}\right)$. Stabilization will occur when $G_{0}^{+}<G_{0}^{-}$, thus for on-axis coinjection, and off-axis counter injection. These combinations of unbalanced NBI have been tested ${ }^{10,28}$ across various machines, and it is seen that the effect on sawteeth is consistent with the effect on the internal kink mode described above. Moreover, the mechanism described by Eq. (19) has been verified against HAGIS (Ref. 18) whereby Eqs. (1) and (2) are evaluated by following guiding centers, and deposited in $\delta W$ with little approximation.

The motivation for the first derivation of an approximated version of Eq. (19) was to explain experiments ${ }^{29}$ in JT-60U showing sawtooth stabilization for strongly tangential beams where the trapped fraction was expected to be small. The beam ions were highly energetic $(350 \mathrm{keV})$ and as such the orbit widths of the passing ions were large. Moreover, since the ions were injected tangentially, it was legiti- 


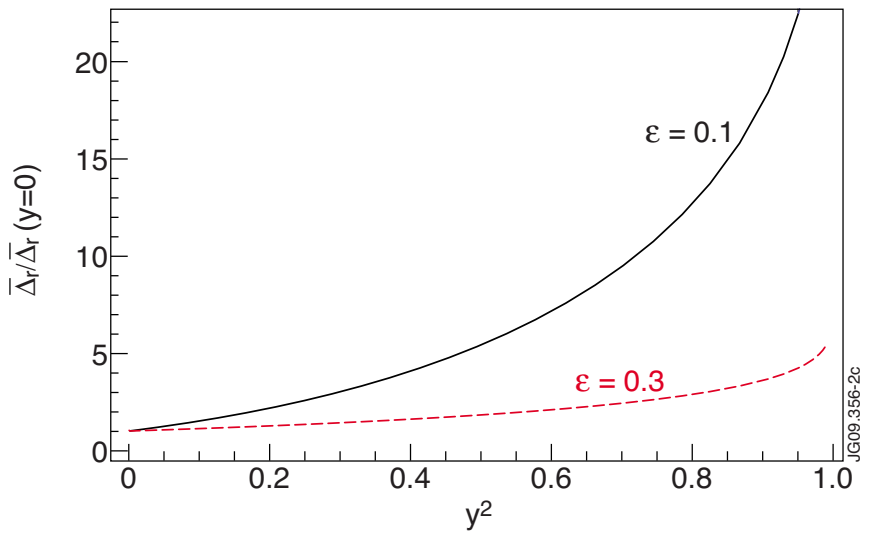

FIG. 2. (Color online) Showing the effective orbit width $\overline{\Delta_{r}}$ normalized to itself with pitch angle $v_{\perp}=0$, plotted with respect to $y$, for two values of $\epsilon$. As usual $y=0$ corresponds to $v_{\perp}=0$ and $y=1$ corresponds to the passingtrapped boundary.

mate to evaluate Eq. (19) for a population with delta distributed pitch angle, for which all particles had the property $v_{\perp}=0$. The calculation showed that the energy of the fast ions had to be large, and the parallel asymmetry strong, in order that the mechanism becomes important. It was therefore initially a surprise that MAST, JET, and TEXTOR experiments (see Ref. 28), which have low energy beam ions, showed that sawteeth were sensitive to the deposition and orientation of beam injection, and that the HAGIS simulations of these pulses proved that the effect on the internal kink mode was due to the finite orbit mechanism described above. The answer to this turns out to be that the orbit width of passing ions becomes larger as the pitch angle approaches the passing-trapped boundary. The orbit width is in fact continuous across the boundary, such that a barely passing ion has the same orbit width as a barely trapped ion. For the issue at hand it can be shown that Eq. (19) is proportional to

$$
\delta W_{r_{1}} \propto \int_{0}^{\infty} d \mathcal{E}(2 \mathcal{E})^{3 / 2} \int_{0}^{1} d y^{2} \sum_{\sigma} G_{0}^{\sigma} \overline{\Delta_{r}},
$$

where $\overline{\Delta_{r}}$ is the velocity and pitch angle weighted effective orbit width is given by

$$
\overline{\Delta_{r}}=\left[\frac{v_{\|}^{2}(\theta=0)}{(2 \mathcal{E})^{3 / 2}}\right] \frac{1}{2 \pi} \int d \theta \cos \theta\left(\frac{R}{R_{0}}\right)\left[\frac{v_{\|}^{2}+v_{\perp}^{2} / 2}{v_{\|}}\right] \Delta_{r} .
$$

In Fig. 2, it is seen that the effective orbit width is strongly enhanced close to the passing-trapped boundary. It is the effective orbit width which determines stability/instability. It is noted now that both high energy particles, and a large barely passing population, is generated with ICRH. Thus it is now clear that we should expect typical ICRH ions to have very large $\bar{\Delta}_{r}$. Moreover, radial gradients in the distribution function tend to be high for ICRH, and parallel velocity asymmetry is created by setting the antenna such that the waves propagate with a preferential toroidal direction. The conditions under which copropagating waves or counterpropagating waves are destabilizing depends sensitively on their radial deposition, as we shall see in the next section.

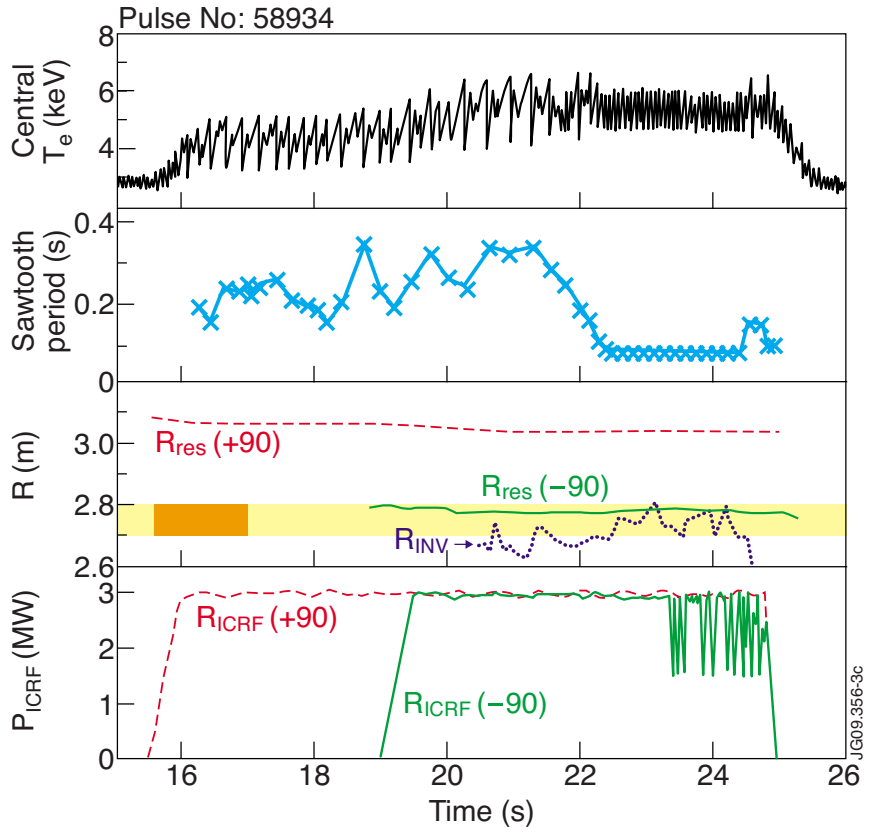

FIG. 3. (Color online) JET Pulse 58934 (Ref. 17), plotting the central electron temperature, sawtooth period, sawtooth inversion radius $R_{\text {inv }}$, and first harmonic $\mathrm{H}$ unshifted cyclotron resonance layers $R_{\text {res }}(\mathrm{H})$ for $+90^{\circ}$ and $-90^{\circ}$ phasings, and heating power for the two antennas. [From J. P. Graves et al., Plasma Phys. Controlled Fusion 47, B121 (2005). Copyright 2005 by Institute of Physics.]

\section{CHARACTERIZING TOROIDALLY PROPAGATING ICRH DISTRIBUTION FUNCTIONS}

In this section, we will concentrate on the key features of the ICRH distribution functions. This will be applied to the well documented $^{17,11}$ JET demonstration discharge 58934. This important discharge demonstrates that an off-axis ion cyclotron resonant wave, with $-90^{\circ}$ phasing (counterpropagating waves), can destabilize (shorten period of) sawteeth even when the sawteeth are initially stabilized by trapped energetic RF ions in the core. Hence, in the latter part of the discharge two resonant ICRH waves coexist. It is the sum of these two populations that ultimately require modeling in order to ascertain the internal kink mode stability. The time trace of the soft $\mathrm{x}$ ray, heating power and unshifted resonance locations, the inversion radius and the sawtooth period of 58934 are reproduced from Ref. ${ }^{30}$ in Fig. 3.

In the following subsections, we aim to develop a model fast ion distribution function that encapsulates the important features of the true distribution function. This then enables analytical calculation of the internal kink mode to be undertaken, and the resulting stability mechanisms to be identified and understood clearly. In any case, within this paper, the analytical results are compared favorably with numerical distribution functions, and with full numerical calculation of the internal kink mode stability.

\section{A. Fast ion density, pressure, and anisotropy}

We will see that the even moments of the distribution function, namely, the density and parallel and perpendicular pressure, depend on the poloidal angle. The reason that the even moments are not flux surface quantities is due to the 
strong anisotropy associated with ICRH. Cyclotron resonance preferentially and locally heats in the perpendicular direction, thus developing trapped orbits with banana tips close to the line of resonance, and hence, localized peaks of pressure and density in the $R-Z$ plane. These effects can be encompassed in a model ${ }^{31}$ of the distribution function, and due to the possibility of obtaining the even moments of the distribution function exactly, the following model has been incorporated in the three-dimensional (3D) equilibrium code VMEC, ${ }^{32}$ and 3D fluid stability code TERPSICHORE. ${ }^{33}$

In this section we require only the leading order distribution $F_{0}$ as defined in Eq. (6). The first finite orbit width correction $F_{1}$ does not affect the even moments, but is required for evaluation of the currents considered in the next section. $F$ is written in terms of a modified bi-Maxwellian which satisfies the lowest order Vlasov equation $\partial F / \partial l=0$ (where $l$ is the arc length along the magnetic field): ${ }^{31}$

$$
\begin{aligned}
F= & \left(\frac{m}{2 \pi e}\right)^{3 / 2} \frac{n_{c}(\bar{r})[1+\sigma c(\bar{r}, \lambda)]}{T_{\perp}(\vec{r}) T_{\|}^{1 / 2}(\vec{r})} \\
& \times \exp \left\{m \mathcal{E}\left[-\frac{\lambda B_{c}}{e T_{\perp}(\vec{r})}-\frac{\left|1-\lambda B_{c}\right|}{e T_{\|}(\vec{r})}\right]\right\},
\end{aligned}
$$

where $\lambda=\mu / \mathcal{E}$ is the pitch, and temperatures are in units of electron volts. The distribution is a modified bi-Maxwellian in the sense that it is not written in terms of $v_{\|}^{2}$ and $v_{\perp}^{2}$. The latter quantities are not constant over the trajectory of a single particle, since they depend in the local value of $B$. The parameter $B_{c}$, is the resonant magnetic field for the ICRH wave. Clearly $A(r) \equiv T_{\perp} / T_{\|}$is a measure of the anisotropy. Finally, $n_{c}$ is related to the particle density, while $\sigma c(r, \lambda)$ is the part of the distribution function that can treat the differing deposition of co- and counterpassing particles. For the even moments of $F_{0}$, the asymmetry is cancelled, and thus $c(r, \lambda)$ does not feature until the treatment of currents in the next section, and finite orbit width corrections to the internal kink mode derived later. By tuning the parameters of Eq. (20), we match the density, parallel and perpendicular temperature, and currents with those of SELFO, and thereby attempt to model the salient features of the distribution function. Clearly, no attempt is made to model far into the energy tail of the distribution function. It should be recognized that the way in which asymmetry enters $F$ (via $\sigma c$ ) is necessarily simple so that the even moments are independent of the asymmetry parameter, and analytical progress can be made in the $\delta W$ calculations. The limitations of Eq. (20) could explain differences observed in Sec. IV between $\delta W_{r_{1}}$ calculated by the analysis and by the SELFO/HAGIS codes.

Taking the zeroth moment of $F_{0}$ yields the variation in the density with respect to $B$ :

$$
n(r, B)=n_{c} N_{B},
$$

where

$$
\begin{aligned}
N_{B}= & \frac{T_{\perp a}}{T_{\perp}} \text { for } B>B_{c} \text { and } \\
N_{B}= & \frac{T_{\perp a}}{T_{\perp}}+\frac{T_{\perp b}-T_{\perp a}}{T_{\perp}}\left(\frac{T_{\perp}}{T_{\|}}\right)^{1 / 2}\left(\frac{B_{c}-B}{B_{c}}\right)^{1 / 2} \\
& \text { for } B<B_{c}, \text { and } \\
T_{\perp a}= & T_{\perp}\left[\frac{B_{c}}{B}+\frac{T_{\perp}}{T_{\|}}\left(1-\frac{B_{c}}{B}\right)\right]^{-1} \text { and } \\
T_{\perp b}= & T_{\perp}\left[\frac{B_{c}}{B}+\frac{T_{\perp}}{T_{\|}}\left(1-\frac{B_{c}}{B}\right)\right]^{-1} .
\end{aligned}
$$

Here we have used

$$
n=\int_{\text {allv }} d v^{3} F_{0}=(1 / 2) \sum_{\sigma} 2 \pi \int_{-\infty}^{\infty} d v_{\|} \int_{-\infty}^{\infty} d v_{\perp} v_{\perp} F_{0} .
$$

Now, taking second moments of the distribution function it can be shown that

$$
P_{\|}=n_{c} T_{\|} H_{\|} \text {and } P_{\perp}=n_{c} T_{\perp} H_{\perp},
$$

where for $B>B_{c}$ :

$$
H_{\|}=\left(\frac{T_{\perp a}}{T_{\perp}}\right) \text { and } H_{\perp}=\left(\frac{T_{\perp a}}{T_{\perp}}\right)^{2},
$$

while for $B<B_{c}$ :

$$
\begin{aligned}
H_{\|}= & {\left[\frac{T_{\perp a}}{T_{\perp}}+\left(\frac{T_{\perp}}{T_{\|}}\right)^{3 / 2}\left(\frac{B_{c}-B}{B_{c}}\right)^{3 / 2}\left(\frac{T_{\perp b}-T_{\perp a}}{T_{\perp}}\right)\right] } \\
H_{\perp}= & {\left[\left(\frac{T_{\perp a}}{T_{\perp}}\right)^{2}+\left(\frac{T_{\perp}}{T_{\|}}\right)^{1 / 2}\left(\frac{B_{c}-B}{B_{c}}\right)^{1 / 2}\right.} \\
& \left.\times\left\{\left(\frac{T_{\perp b}-T_{\perp a}}{2 T_{\perp}}\right)\left(\frac{B}{B_{c}}\right)+\frac{T_{\perp b}^{2}-T_{\perp a}^{2}}{T_{\perp}^{2}}\right\}\right] .
\end{aligned}
$$

Here, 3D data files from the SELFO simulations of $n, P_{\perp}$, and $P_{\|}$are used to identify the three radially dependent parameters of the model distribution function. In particular, in the following, the left-hand side of the equations correspond to the parameters in the model, and the right-hand side of the equations are the quantities from the SELFO simulations:

$$
\begin{aligned}
& n_{c}(r)=n\left(R_{c}, Z\right), T_{\perp}(r)=\frac{P_{\perp}\left(R_{c}, Z\right)}{e n\left(R_{c}, Z\right)} \text { and } \\
& A(r) \equiv \frac{T_{\perp}(r)}{T_{\|}(r)}=\frac{P_{\perp}\left(R_{c}, Z\right)}{P_{\|}\left(R_{c}, Z\right)},
\end{aligned}
$$

where $R_{c}$ is the RF minority resonance major radius such that $B_{c}=B\left(R_{c}\right)$. Now, since the heating is approximately located on a vertical line through the plasma cross section we can resolve the minor radius on the left-hand side of the equations through $r^{2}=Z^{2}+\left(R_{c}-R_{0}\right)^{2}$ with $Z$ defining the distance along a vertical chord $R=R_{c}$. Here a circular cross section has been assumed, which is consistent with SELFO.

Where there are two resonant surfaces the problem is treated upon assuming the sum of model distributions. This 

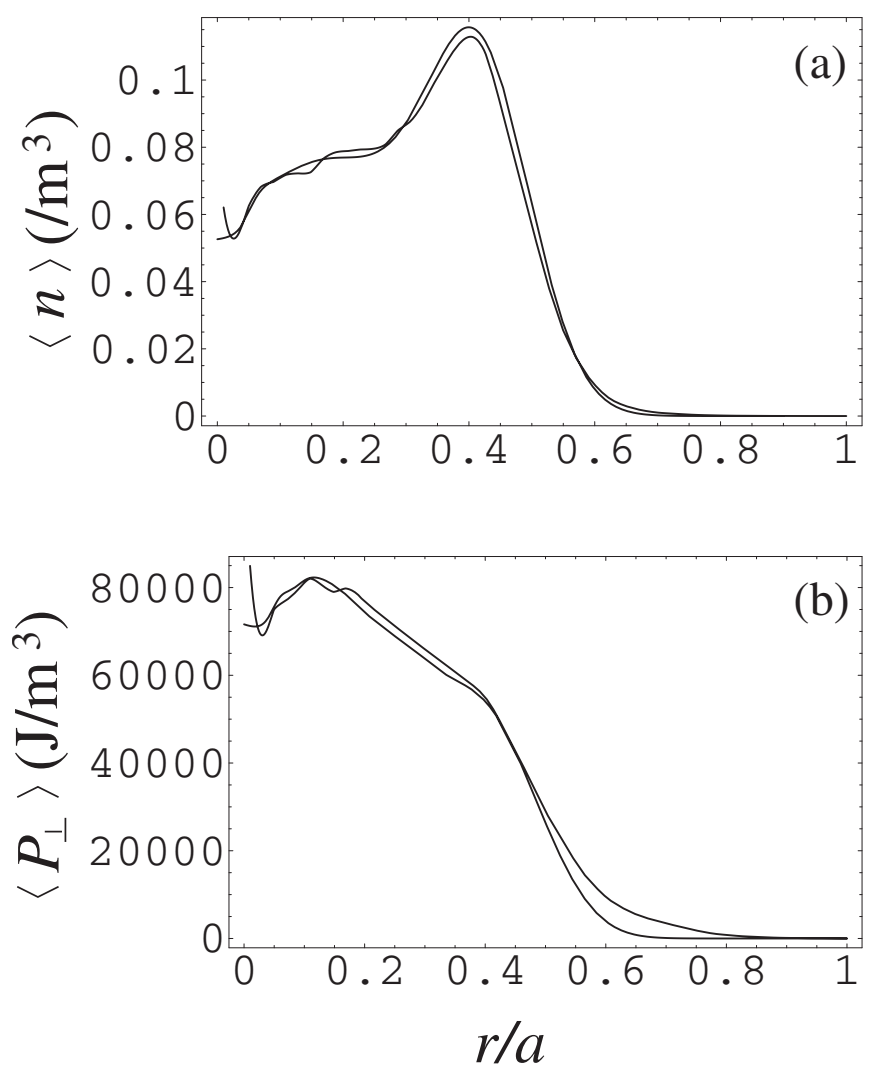

FIG. 4. Showing the flux averaged density (units of $10^{19}$ ) and perpendicular pressure as a function of normalized minor radius. Comparisons between the profiles from the SELFO code, and the flux averages of Eqs. (21) and (22), for the latter part of JET discharge 58934.

is a reasonable assumption because RF-wave-particle interaction is strongest for trapped particles bouncing on a resonant layer. If the width of separation of the two resonances is not less than the radial excursion of a fast ion, then a particle bouncing on one resonance will only be weakly affected by the other. Hence there are now six radially dependent parameters to resolve, namely, $n_{c}(r), T_{\perp}(r)$, and $A(r)$ for the two distributions. The problem has been treated upon exploitation of simulations with off-axis heating alone. This enabled identification of the three functions for off-axis heating, and thus when employed in conjunction with the parameters obtained with on-axis heating alone, provided a first guess for the distribution function for the combined heating case. The six functions were then normalized iteratively to provide a best parameter fit of the 3D plots of the density, parallel pressure and perpendicular pressure. The result of such a procedure for the case at hand, i.e., JET discharge 58 934, has been undertaken, and one finds, for example, that the anisotropy $A(r)$ is of order of ten. The resulting flux surfaced averaged density and parallel pressure, and compared with the SELFO data in Fig. 4 for the latter part of discharge 58934 when both RF antennas are being deployed. Finally, Fig. 5 shows the density over the entire poloidal cross section. Peaks in the density result from the localized deposition of the RF heating (resonances shown with near vertical lines), and again recover the salient features of the SELFO data.

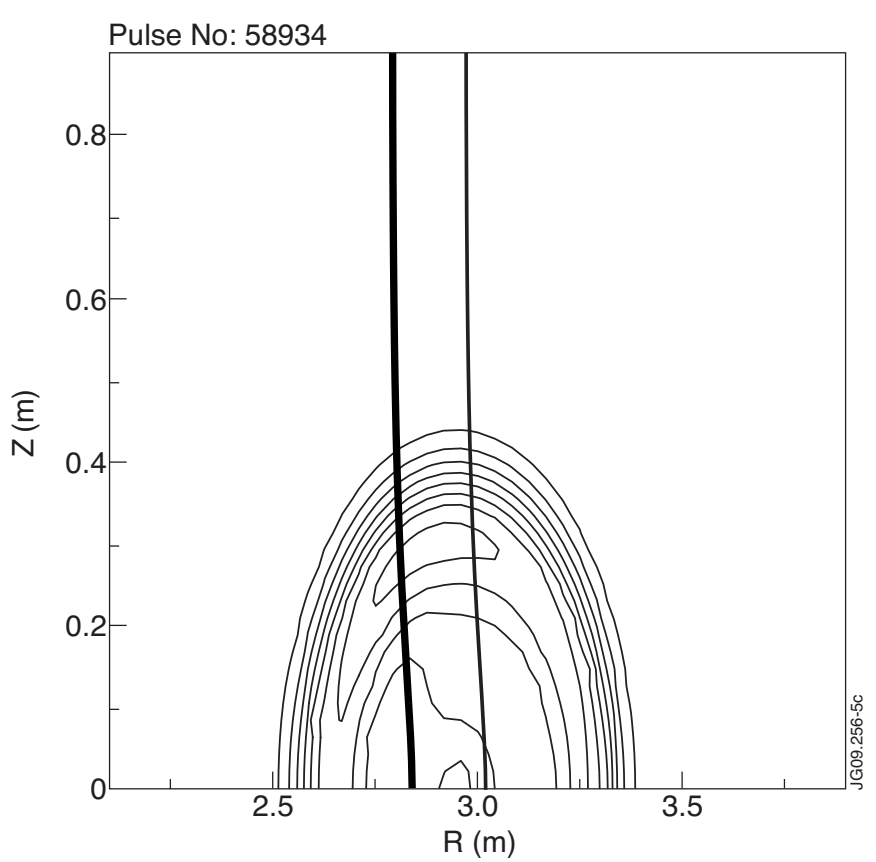

FIG. 5. Showing the fast ion density across the $R-Z$ plane according to Eq. (21) for the latter part of 58934 . The anisotropic distribution function recovers the local peaks in density, which are due to an abundance of trapped ions along lines tangent to the resonances. The location of the resonant field strengths are indicated with near vertical lines.

\section{B. Currents in asymmetric fast ion distributions}

The parallel velocity asymmetry is conveniently visualized through the parallel current density $j_{\|}=e Z \int d v^{3} v_{\|} F$. We derive the currents for a general distribution function, and then specialize to our model distribution of Eq. (20). First we define $F^{+}$as the distribution function of particles moving in the same direction as the plasma current, and $F^{-}$as the distribution counter to the plasma current. It is more conventional to discuss current drive in terms of the toroidal current density $j_{\phi}=e Z \int d v^{3} v_{\phi} F$ than the parallel current density. In the following it is the parallel current that is evaluated analytically, but we label the currents as toroidal. Moreover, SELFO evaluates the toroidal component of the parallel current. In any case the correction is much smaller than the currents described below. We recall the definitions of $F_{0}$ and $G_{0}$ in Eqs. (6) and (7), to reveal the orbit width expansion of the current $j_{\phi}=j_{\phi 0}+j_{\phi 1}$, where

$$
j_{\phi_{0}}=Z e \pi \int_{0}^{\infty} d \mathcal{E}(2 \mathcal{E}) \int_{0}^{1 / B_{\max }} d \lambda B\left(F_{0}^{+}-F_{0}^{-}\right)
$$

and

$$
\begin{aligned}
j_{\phi_{1}}= & -Z e \pi \int_{0}^{\infty} d \mathcal{E}(2 \mathcal{E}) \int_{0}^{1 / B} d \lambda B \frac{q}{r \Omega_{c}}\left(\left|v_{\|}\right| R-p R_{0}^{2} q \omega_{b}\right) \\
& \times\left(G_{0}^{+}+G_{0}^{-}\right),
\end{aligned}
$$

with $p=1$ for passing particles, $p=0$ for trapped particles, and $\Omega_{c}=e Z B_{0} / m$. Note that we have used the result for large aspect ratio circular geometry $\Delta_{r}=q\left[v_{\|} R\right.$ $\left.-R_{0}^{2} q(p \sigma) 2 \pi / \tau_{b}\right] /\left(r \Omega_{c}\right)$, valid for both trapped and passing 
particles, but where $\tau_{b}=2 \pi / \omega_{b}$ is the transit time for passing ions.

We note now that $j_{\phi 0}$ is valid only for passing particles, and hence the limit of integration $\lambda=1 / B_{\max }$. Due to the poloidal reflection of trapped particles, there is no current associated with trapped particles at that order, and hence for trapped ions $F_{0}^{+}-F_{0}^{-}=0$. We note that $j_{\phi_{0}}$ can encompass both Fisch currents, ${ }^{16}$ and the currents associated with detrapping into preferentially co- or counterpassing ions. ${ }^{34,11}$ In this paper we do not differentiate between which of these mechanisms creates $j_{\phi_{0}}$, but we note that both are consistent with an asymmetric distribution in $v_{\|}$at the lowest order, i.e., in $F_{0}$. The finite orbit corrected current $j_{\phi_{1}}$ is essentially independent of asymmetry in the lowest order distribution function, and we see that any distribution of particles have currents associated with constraint of canonical momentum conservation. It is seen here that $j_{\phi_{1}}$ is important when the radial gradient of the distribution is large, and when the orbit widths of the particles are large. Hence, these currents are seen as the drift orbit analog of finite Larmor radius diamagnetic currents. It is clear then that $j_{\phi_{1}}$ can be large for ICRH because the ions are heated to high energy, and ions are pushed toward trapped and deeply trapped pitch angles, and also the local nature of ICRH can create large radial gradients in the distribution function. Finally, barely passing ions contribute to a small orbit current, $j_{\phi_{1}}$, while, due to their small orbit width, deeply passing ions hardly contribute to $j_{\phi_{1}}$ at all. Finally, we note that barely passing fast ions also contribute to a bootstrap current, ${ }^{35}$ despite being neglected in the above definition of $j_{\phi_{1}}$. However, since pitch angle scattering is very weak for energetic ions, the fast ion bootstrap current is of order $\epsilon$ smaller than the bootstrap current associated with electrons (given a like for like pressure profile). ${ }^{35}$ Resultingly, the fast ion bootstrap current can be ignored. To summarize, trapped ions cannot contribute to the current due to parallel asymmetry, $j_{\phi_{0}}$, but meanwhile trapped ions strongly dominate the finite orbit width current $j_{\phi_{1}}$. Consequently, providing the currents are not dominated by the effects of nonstandard orbits, one can approximately identify $j_{\phi_{0}}$ with the total current from passing ions, and $j_{\phi_{1}}$ with the total current from trapped ions.

For the internal kink mode analysis and the interpretation that follows, it is necessary to be able to identify separately $j_{\phi_{0}}$ and $j_{\phi_{1}}$, since the mechanism determining sawtooth destabilization due to local ICRH deposition is found to be dependent on asymmetric distribution functions in $v_{\|}$at the lowest order, and thus must be consistent with having finite $j_{\phi_{0}}$. Since it is the total fast ion current $j_{\phi}$ that is either measured or simulated (e.g., using SELFO), we can estimate $j_{\phi_{0}}$ and hence the parallel asymmetries in the distribution function $F_{0}^{+}-F_{0}^{-}$, from $j_{\phi}-j_{\phi_{1}}$, where, as we have mentioned $j_{\phi_{1}}$ is not sensitive to the asymmetry in the number of coand counterpassing ions. In practice however, as mentioned above, providing the currents are not dominated by the effects of nonstandard orbits, one can approximately identify $j_{\phi_{0}}$ with the total current from passing ions, and $j_{\phi_{1}}$ with the total current from trapped ions.
We now undertake to calculate the flux averaged currents for our model distribution function, parametrized with the SELFO simulations of JET discharge 58 934. The critical resonant field $B_{c}$ is written in terms of $\epsilon_{c}=\left(R_{c}-R_{0}\right) / R_{0}$, so that $B_{c} \approx B_{0}\left(1-\epsilon_{c}\right)$. Hence for the on-axis resonant wave of 58934, $\epsilon_{c} \approx 0$, while for the off-axis resonant wave, $\epsilon_{c} \approx-0.3 m / R_{0}$, with $R_{0} \approx 3 m$. Writing the temperature in units of electron volts, we have upon employing the model of Eq. (20),

$$
\begin{aligned}
\left\langle j_{\phi 0}(r)\right\rangle= & \operatorname{Zen}_{c}\left(\frac{2 e T_{\perp} A}{\pi m}\right)^{1 / 2} \\
& \times \int_{0}^{1} d y^{2} \frac{2 \epsilon c\left(r, y^{2}\right)}{\left[y^{2}\left(1-\epsilon_{c}\right)+A\left|2 \epsilon-y^{2}\left(\epsilon-\epsilon_{c}\right)\right|\right]^{2}},
\end{aligned}
$$

where, from the model distribution function of Eq. (20), we recall that $n_{c}(r) c(r, y)$ is the asymmetric contribution to the density. Due to the requirement that mirror trapped particles cannot contribute to $j_{\phi 0}$, and if the distribution function is to be continuous across the passing-trapped boundary, we have the condition $c=0$ at the passing-trapped boundary, i.e., $c\left(r, y^{2}=1\right)=0$.

The finite orbit corrections must be broken down into trapped and passing, denoted by superscript $t$ and $p$, respectively, so that employing the model of Eq. (20),

$$
\begin{aligned}
\left\langle j_{\phi 1}(r)\right\rangle^{p}= & -e\left(\frac{3}{2}\right)\left(\frac{R_{0} q}{r B_{0}}\right) \\
& \times \int_{0}^{1} d y^{2} \frac{(2 \epsilon)^{3 / 2}}{\left[y^{2}\left(1-\epsilon_{c}\right)+A\left|2 \epsilon-y^{2}\left(\epsilon-\epsilon_{c}\right)\right|\right]^{5 / 2}} \\
& \times\left[\left(n_{c} T_{\perp} A^{1 / 2}\right)^{\prime}-\left(\frac{5}{2}\right)\right. \\
& \left.\times \frac{\left(n_{c} T_{\perp} A^{1 / 2}\right) A^{\prime}\left|2 \epsilon-y^{2}\left(\epsilon-\epsilon_{c}\right)\right|}{y^{2}\left(1-\epsilon_{c}\right)+A\left|2 \epsilon-y^{2}\left(\epsilon-\epsilon_{c}\right)\right|}\right] J^{p}\left(r, y^{2}\right),
\end{aligned}
$$

and for trapped particles,

$$
\begin{aligned}
\left\langle j_{\phi 1}(r)\right\rangle^{t}= & -e\left(\frac{3}{2}\right)\left(\frac{R_{0} q}{r B_{0}}\right) \\
& \times \int_{0}^{1} d k^{2} \frac{(2 \epsilon)^{3 / 2}}{\left[1-\epsilon_{c}+A\left|2 \epsilon k^{2}-\left(\epsilon-\epsilon_{c}\right)\right|\right]^{5 / 2}} \\
& \times\left[\left(n_{c} T_{\perp} A^{1 / 2}\right)^{\prime}-\left(\frac{5}{2}\right)\right. \\
& \left.\times \frac{\left(n_{c} T_{\perp} A^{1 / 2}\right) A^{\prime}\left|2 \epsilon k^{2}-\left(\epsilon-\epsilon_{c}\right)\right|}{1-\epsilon_{c}+A\left|2 \epsilon k^{2}-\left(\epsilon-\epsilon_{c}\right)\right|}\right] J^{t}\left(r, k^{2}\right),
\end{aligned}
$$

where $X^{\prime} \equiv d X / d r, \epsilon=r / R_{0}$, and we have 

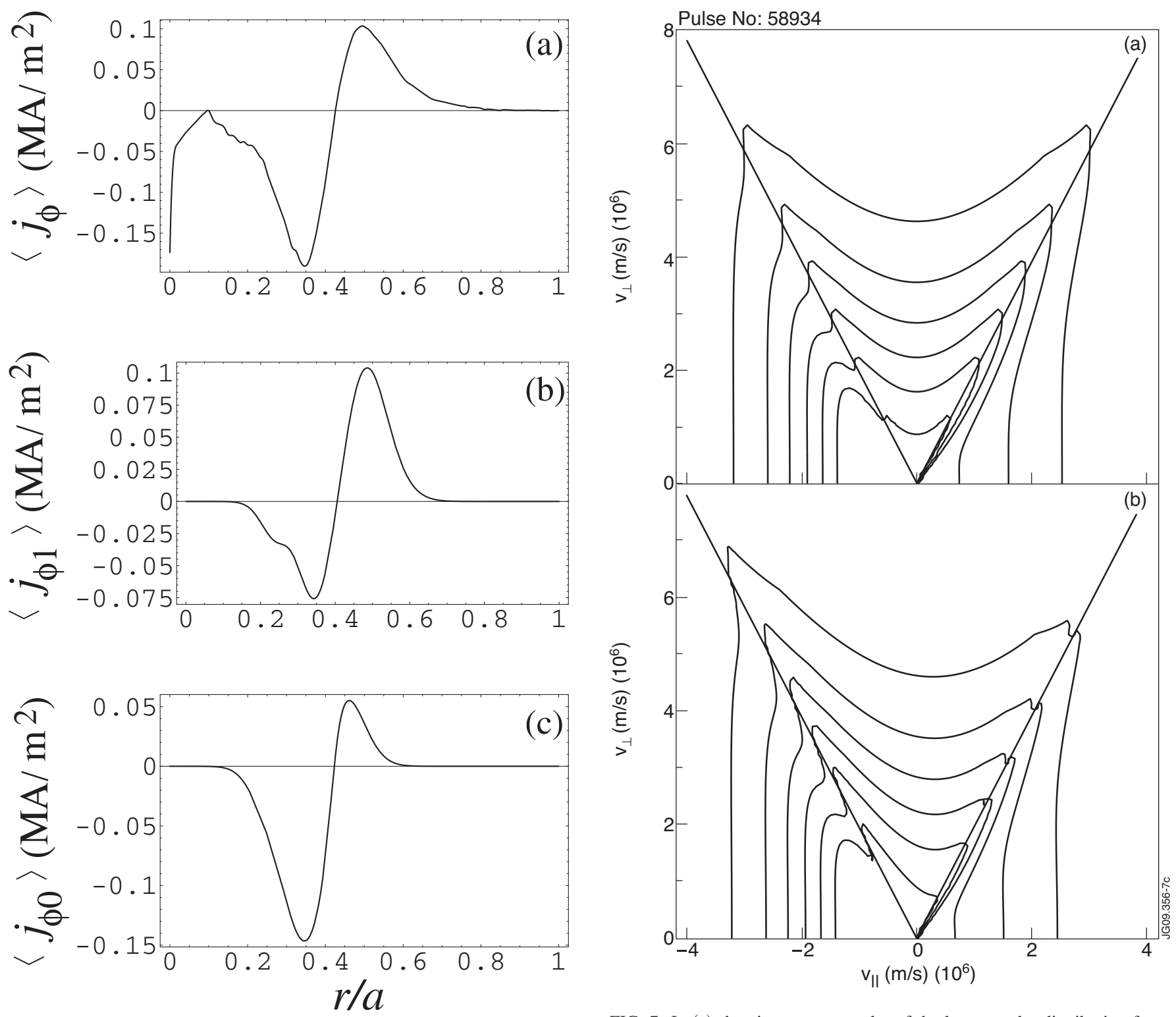

FIG. 6. Showing (a) the SELFO deduced flux averaged current density $\left\langle j_{\phi}(r)\right\rangle$ (after plasma drag has been deducted) as a function of minor radius for discharge 58 934. (b) Plots finite orbit currents $\left\langle j_{\phi 1}(r)\right\rangle$, the sum of Eqs. (24) and (25), while (c) plots the current due to the asymmetries in the lowest order distribution function $\left\langle j_{\phi 0}(r)\right\rangle$, and is obtained by subtracting the currents shown in (b) from (a) multiplied by $j_{d}^{-1}$. [Figure partially reproduced from J. P. Graves, I. T. Chapman, S. Coda, L.-G. Eriksson, and T. Johnson, Phys. Rev. Lett. 102, 065005 (2009). Copyright 2009 by American Physical Society.]

$$
J^{t}=\frac{2}{\pi} E\left(k^{2}\right)+\frac{k^{2}-1}{\pi} K\left(k^{2}\right) \text { and } J^{p}=\frac{2}{\pi} E\left(y^{2}\right)-\frac{\pi}{2 K\left(y^{2}\right)},
$$

where $K$ and $E$ are complete elliptic integrals of the first and second kinds, respectively.

Figure 6(a) shows the SELFO deduced flux averaged current density $\left\langle j_{\phi}(r)\right\rangle$ as a function of minor radius for discharge 58 934. We note that the SELFO current has been obtained by evaluating the parallel current from fast ion distribution function, and subtracting the drag current from the background plasma. Figure $6(\mathrm{~b})$ plots $\left\langle j_{\phi 1}(r)\right\rangle$, the sum of Eqs. (24) and (25), which are the currents due to finite drift

FIG. 7. In (a) showing a contour plot of the lowest order distribution function $F_{0}$, according to Eqs. (20) and (6), while in (b) plotting the more accurate $F \approx F_{0}-\Delta r G_{0}$, with $G_{0}$ given by Eq. (7), all for discharge 58934 .

orbits. These currents are now straightforward to calculate because $T_{\perp}(r), n_{c}(r)$, and $A(r)$ were obtained in Sec. III A for the two distributions. We note here that $\left\langle j_{\phi 1}(r)\right\rangle^{t}$ is found to be at least an order of magnitude larger than $\left\langle j_{\phi 1}(r)\right\rangle^{p}$. Since the currents from passing ions are found to be the same order of magnitude as trapped ions. ${ }^{11}$ This means that the majority of the current attributed to passing ions must be contained in $\left\langle j_{\phi 0}(r)\right\rangle$ (note that this statement may need to be relaxed if the passing ion currents are dominated by the effects of nonstandard orbits). The flux averaged current $\left\langle j_{\phi 0}(r)\right\rangle$ due to the asymmetries in the lowest order distribution function $F_{0}$, is identified with $\left\langle j_{\phi}(r)\right\rangle-\left\langle j_{\phi 1}(r)\right\rangle$. It is obtained by multiplying the SELFO current of Fig. 6(a) by the inverse of the plasma drag coefficient [i.e., by $1 / j_{d}$, where $j_{d}$ is defined in Eq. (30)], and then to subtract from this $\left\langle j_{\phi 1}(r)\right\rangle$, i.e., the current of Fig. 6(b). Figure 6(c) shows $\left\langle j_{\phi 0}(r)\right\rangle$ after it has been smoothed.

Nevertheless, knowledge of the full distribution func- 
tion, and development of the internal kink analysis in the next subsection, requires the asymmetry function $c\left(r, y^{2}\right)$ be resolved. Identification of the latter requires further modeling. We let $c=c_{r}(r) c_{y}\left(y^{2}\right)$, and choose $c_{y}$ to be log-normal in $1-y^{2}$. This enables the lowest order asymmetry to disappear at the passing-trapped boundary as required. Hence, setting

$$
c_{y}\left(y^{2}\right)=\frac{5}{4} \frac{1}{1-y^{2}} \exp \left[-\left(\ln \left\{\frac{5}{8}\left(1-y^{2}\right)\right\}\right)^{2}\right]
$$

we can now resolve $c_{r}(r)$ and hence $c\left(r, y^{2}\right)$, and thus the entire distribution function. Shown in Fig. 7(a) is a contour plot of $F_{0}$ given by Eqs. (20) and (6), i.e., the distribution function in absence of finite orbit corrections, plotted with respect to $v_{\|}$and $v_{\perp}$ on the outboard side $(\theta=0)$ and at $r / a=0.35$. Features shown in Fig. 7(a) include the peaking of the distribution function on the trapped side of the passingtrapped boundary, which is due to an abundance of trapped particles along the line of the off-axis RF resonance, and also the asymmetry in $v_{\|}$which is consistent with $c\left(r, y^{2}\right)$ and the lowest order current $\left\langle j_{\phi 0}(r)\right\rangle$ at $r / a=0.35$ shown in Fig. 6(c).
Finally, Fig. 7(b) shows $F_{0}-\Delta r G_{0} \approx F$ given by Eqs. (20), (6), and (7), i.e., the total distribution including the effects of finite orbit widths, plotted with respect to $v_{\|}$and $v_{\perp}$ on the outboard side $(\theta=0)$ and at $r / a=0.35$. We now see additional asymmetries in $v_{\|}$, particularly inside the trapped cone. The corresponding currents are consistent with the total current $\left\langle j_{\phi}(r)\right\rangle$ at $r / a=0.35$ shown in Fig. 6(a).

\section{Internal kink stability for JET minority H demonstration discharge}

In this section, we will develop the internal kink stability in terms of the model distribution function given by Eq. (20), and compare results with the solution to the fast ion $\delta W$ given by the HAGIS code for the full distribution function obtained by SELFO. The results will be compared with the main features of the relevant experiment.

For the semianalytical treatment, it just remains to calculate $D_{t}^{+}+D_{t}^{-}, D_{p}^{+}+D_{p}^{-}$, and $C^{+}-C^{-}$for $F_{0}$ given by Eq. (20). This is easily achieved:

$$
\begin{aligned}
& D_{p}^{+}+D_{p}^{-}=\frac{\frac{d}{d r}\left(n_{c} e T_{\perp} A^{1 / 2}\right)-\left(\frac{5}{2}\right) \frac{d A}{d r}\left[\frac{\left(n_{c} e T_{\perp} A^{1 / 2}\right)\left|2 \epsilon-y^{2}\left(\epsilon-\epsilon_{c}\right)\right|}{y^{2}\left(1-\epsilon_{c}\right)+A\left|2 \epsilon-y^{2}\left(\epsilon-\epsilon_{c}\right)\right|}\right]}{\left[y^{2}\left(1-\epsilon_{c}\right)+A\left|2 \epsilon-y^{2}\left(\epsilon-\epsilon_{c}\right)\right|\right]^{5 / 2}}, \\
& D_{t}^{+}+D_{t}^{-}=\frac{\frac{d}{d r}\left(n_{c} e T_{\perp} A^{1 / 2}\right)-\left(\frac{5}{2}\right) \frac{d A}{d r}\left[\frac{\left(n_{c} e T_{\perp} A^{1 / 2}\right)\left|2 \epsilon k^{2}-\left(\epsilon-\epsilon_{c}\right)\right|}{1-\epsilon_{c}+A\left|2 \epsilon k^{2}-\left(\epsilon-\epsilon_{c}\right)\right|}\right]}{\left[1-\epsilon_{c}+A\left|2 \epsilon k^{2}-\left(\epsilon-\epsilon_{c}\right)\right|\right]^{5 / 2}},
\end{aligned}
$$

and

$$
C^{+}-C^{-}=e T_{\perp}(r)\left\{\frac{\frac{\partial}{\partial r}\left[n_{c} A^{1 / 2} c\left(r, y^{2}\right)\right]-n_{c} A^{1 / 2}\left(2-y^{2}\right)\left(\frac{y^{2}}{2}\right) \frac{\partial}{\partial y^{2}} c\left(r, y^{2}\right)-3 \frac{d A}{d r}\left[\frac{\left(n_{c} A^{1 / 2} c\left(r, y^{2}\right)\right)\left|2 \epsilon-y^{2}\left(\epsilon-\epsilon_{c}\right)\right|}{y^{2}\left(1-\epsilon_{c}\right)+A\left|2 \epsilon-y^{2}\left(\epsilon-\epsilon_{c}\right)\right|}\right]}{\left[y^{2}\left(1-\epsilon_{c}\right)+A\left|2 \epsilon-y^{2}\left(\epsilon-\epsilon_{c}\right)\right|\right]^{3}}\right\},
$$

where we recall that $A=T_{\perp} / T_{\|}$and $c$ are dimensionless, so that $D_{t}^{+}+D_{t}^{-}, D_{p}^{+}+D_{p}^{-}$, and $C^{+}-C^{-}$are seen to be pressure gradients weighted appropriately to account for the single particle dynamics concerned.

It is now straightforward to evaluate $\delta W$ for the fast ion distribution function of JET discharge 58934. It is necessary to take into account $D_{t}^{+}+D_{t}^{-}, D_{p}^{+}+D_{p}^{-}$, and $T_{\perp}^{1 / 2}\left(C^{+}-C^{-}\right)$for both of the two fast ion populations, corresponding to resonance with the two ICRH waves. The resulting $\delta W$ contributions are then added together and compared with ideal and resistive stability criteria. In order to demonstrate the sensitivity of $\delta W$ to the position of the current drive, and hence the position of the off-axis resonance, we instead allow $r_{1}$ to move relative to fixed off- and on-axis resonance locations. This then also allows us to compare with HAGIS simulations, which take the full marker distribution from SELFO, and calculates the internal kink mode stability over a range of $r_{1}$. The results of the analytical calculation are shown in Fig. 8.
The dot-dash curve shows the conventional fast ion contribution $\delta \hat{W}_{0}=\delta \hat{W}_{k t}+\delta \hat{W}_{f t 0}+\delta \hat{W}_{f p 0}$ given by the sum of Eqs. (10), (14), and (15), which yields the effect of fast ions on stability in the absence of finite orbit effects, and is plotted as a function of $r_{1}$. The magnetic shear, as well as all plasma and fast ion related profiles remain fixed. It is seen that as $r_{1}$ increases, $\delta \hat{W}_{0}$ increases, and this result is consistent with (roughly) the dependence of $\int_{0}^{r_{1}} d r r^{3 / 2}\left(-P_{h}^{\prime}\right)$ on $r_{1}$. The solid line in Fig. 8 plots the finite orbit correction of $\delta \hat{W}_{r_{1}}$, given by Eq. (19), as a function of $r_{1}$. It is seen that there is a deep and narrow minimum in $\delta \hat{W}_{r_{1}}$ close to $r_{1} / a=0.41$, which is close to the measured inversion radius of $r_{\text {inv }} / a=0.34$. As we will see, the large and negative $\delta \hat{W}$ at $r_{1} / a=0.41$ would make the internal kink mode ideally unstable, and is thus consistent with the very small sawteeth observed in discharge 58934.

Let us examine in more detail the physics behind the 


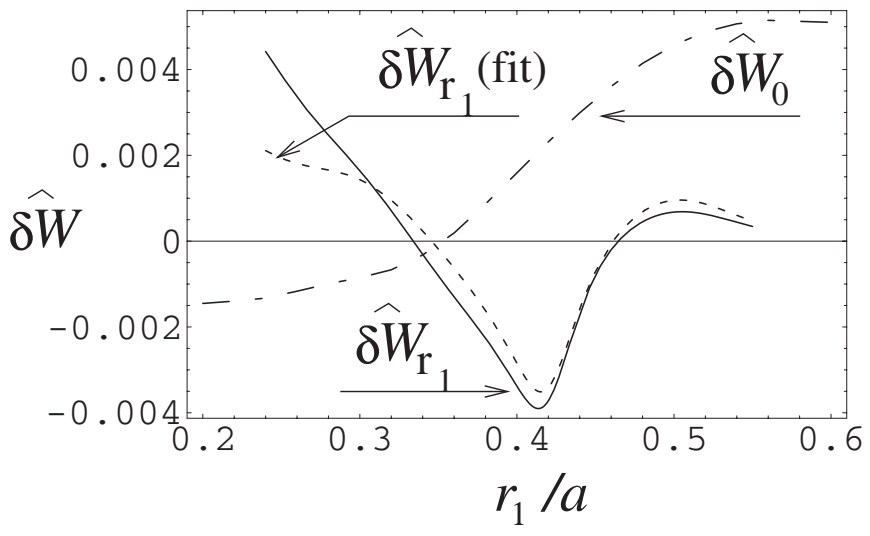

FIG. 8. $\delta \hat{W}_{0}=\delta \hat{W}_{k t}+\delta \hat{W}_{f t 0}+\delta \hat{W}_{f p 0}$ and $\delta \hat{W}_{r 1}$ according to Eq. (19), and also $\delta \hat{W}_{r 1}$ according to the fit of Eq. (29), plotted as a function of $r_{1}$, for discharge 58 934. [Figure reproduced from J. P. Graves, I. T. Chapman, S. Coda, L.-G. Eriksson, and T. Johnson, Phys. Rev. Lett. 102, 065005 (2009). Copyright 2009 by American Physical Society.]

extreme sensitivity of $\delta \hat{W}_{r_{1}}$ to the location of $r_{1}$. Simply by inspection of Eqs. (23), (19), and (28), it is clear that $\delta \hat{W}_{r_{1}}$ and the radial derivative of $j_{\phi 0}$ are related. We recall that the current associated with energetic passing ions, in the absence of drag, is almost entirely encapsulated in $j_{\phi 0}$, since $j_{\phi 1}$ is dominated by trapped ions. This means that we should be able to determine the stability of the internal kink mode relative to the passing ion current profile prior to the deduction of the plasma drag current. In fact, some simple algebra and numerical integration over pitch angle $y^{2}$ reveals the following fit:

$$
\delta \hat{W}_{r_{1}} \approx-\left.\frac{2^{1 / 2}}{\pi \epsilon_{1}^{2}} \frac{1}{Z \Omega_{c}}\left(\frac{2 \mu_{0}}{B_{0}^{2}}\right) T_{\perp}^{1 / 2} T_{\|}^{1 / 2} \frac{d}{d r}\left\langle j_{\phi 0}\right\rangle\right|_{r_{1}} .
$$

The dashed line of Fig. 8 plots Eq. (29), for the current profile of Fig. 7(a), as a function of $r_{1}$, and it seen that there is excellent agreement with Eq. (19). It is therefore clear that maximum instability occurs when the largest positive gradient in the fast ion current dipole coincides with the location of $r_{1}$. The location of the maximum gradient in $j_{\phi 0}$ is close to the Doppler shifted resonance position, corresponding also closely to the zero in $j_{\phi 0}$. Furthermore, we recall that one can approximately identify $j_{\phi 0}$ with the entire passing ion current prior to the deduction of the plasma drag current, and hence we can now directly obtain an approximation for $\delta \hat{W}_{r_{1}}$ following calculation of the passing ion current profile such as those obtained by SELFO [see e.g., Fig. 19(a) of Ref. 11].

These new calculations and derivations in this manuscript provide an answer to why toroidally propagating RF waves are so successful at sawtooth control despite only having a modest effect on the magnetic shear. In particular, for JET discharge 58 934, SELFO simulations demonstrate that the effect of the fast ion current profile leads to a maximum change in the shear of only 0.1 . The corresponding change in the ideal or resistive internal kink threshold, in the absence of the finite orbit effect of Eq. (29), is small. It is in fact clear that the classical mechanism involving only the shear at $q=1$ is dwarfed by the fast ion mechanism described here.

\section{MINORITY ${ }^{3}$ HE SAWTOOTH EXPERIMENTS AND SIMULATIONS}

In order to clearly show that the fast ion mechanism is responsible for sawtooth control it is desirable to attempt to reduce the effect that fast ions have on the magnetic shear. While for the hydrogen minority pulse analyzed above the change in the shear due to ions was around 0.1 , the change in the shear arising from minority ${ }^{3} \mathrm{He}$ is much smaller. This subsection summarizes dedicated experiments employing minority ${ }^{3} \mathrm{He}$, with resonance placed on the high field side close to $r_{1}$. The details of these pulses, and other more advanced pulses, have been published elsewhere. ${ }^{36}$

As mentioned already, the experimental objective of generating negligible net minority ion current in the core is made realizable upon choosing minority ${ }^{3} \mathrm{He}$. The fast ion current density $j_{h}=e n_{h} Z_{h} v_{h}$ can be evaluated by SELFO, where $v_{h}$ is the $v_{\|}$moment of the distribution function. However, the plasma is dragged along with the fast ions, such that the total current is proportional to a drag coefficient $j_{d}$ such that $j_{\text {tot }}=j_{h} \times j_{d}$. The fast ion current is subject to momentum conservation, quasineutrality and the balance of collision rates of electrons on all ion species, ${ }^{16}$ giving

$$
\begin{aligned}
j_{d}= & 1-\left\{\frac{Z_{h}}{Z_{\text {eff }}}+\frac{m_{h} \Sigma_{i} Z_{i} n_{i}\left[1-\left(Z_{i} / Z_{\text {eff }}\right)\right]}{Z_{h} \Sigma_{i} n_{i} m_{i}}\right. \\
& \left.-G\left(\frac{Z_{h}}{Z_{\text {eff }}}-\frac{m_{h} \Sigma_{i} n_{i} Z_{i}^{2}}{Z_{h} Z_{\text {eff }} \Sigma_{i} n_{i} m_{i}}\right)\right\},
\end{aligned}
$$

where $G=1.46 A\left(Z_{e f f}\right) \epsilon^{1 / 2}, A$ is a weak function of $Z_{e f f}$, and $i$ denotes ion species other that hot $(h)$. Due to the minority ion mass number $m_{h}=3$ and charge $Z_{h}=2$ and moderate $Z_{\text {eff }} \approx 1.8$ giving $A \approx 1.4$, the effect of the plasma drag is to reverse the sign of the net current density inside $q=1$, and to neutralize the current density and the change in the shear at $q=1$.

The objective of the experiment was for the ${ }^{3} \mathrm{He}$ resonance to pass slowly through the inversion radius on the high field side in each discharge. This was technically difficult, because the fundamental hydrogen resonance needed to remain outside the antenna region $(>4 m)$ at all times. A particular configuration was chosen which permitted the ${ }^{3} \mathrm{He}$ resonance to access a $q=1$ radius which was not compromised in size. The two pulses summarized in Fig. 9 had the slowest field and current ramp, and the clearest sawtooth control signatures. The field was varied from $2.9 T$ to $2.96 T$. The pulses were identical, except, crucially, $76189 \mathrm{em}-$ ployed $3 \mathrm{MW}$ of counterpropagating waves $\left(-90^{\circ}\right)$, while 76190 employed $2 \mathrm{MW}$ of copropagating waves $\left(+90^{\circ}\right)$. Also shown in Fig. 9 is the NBI power, the core central electron temperature, the sawtooth period, and the $n=1 \mathrm{mag}$ netics amplitude for both pulses. All of these signals show the contrasting effects of the antenna phasing on the sawteeth (and internal kink instability in the case of the magnetics signal).

The minority ion concentration was around $1 \%$, giving fast ion tail temperatures in excess of $250 \mathrm{keV}$. Sawteeth were strongly affected when the resonance was about $2-6 \mathrm{~cm}$ inside the inversion radius $\left(r_{\text {inv }}\right)$. Discharge $76189\left(-90^{\circ}\right)$ 


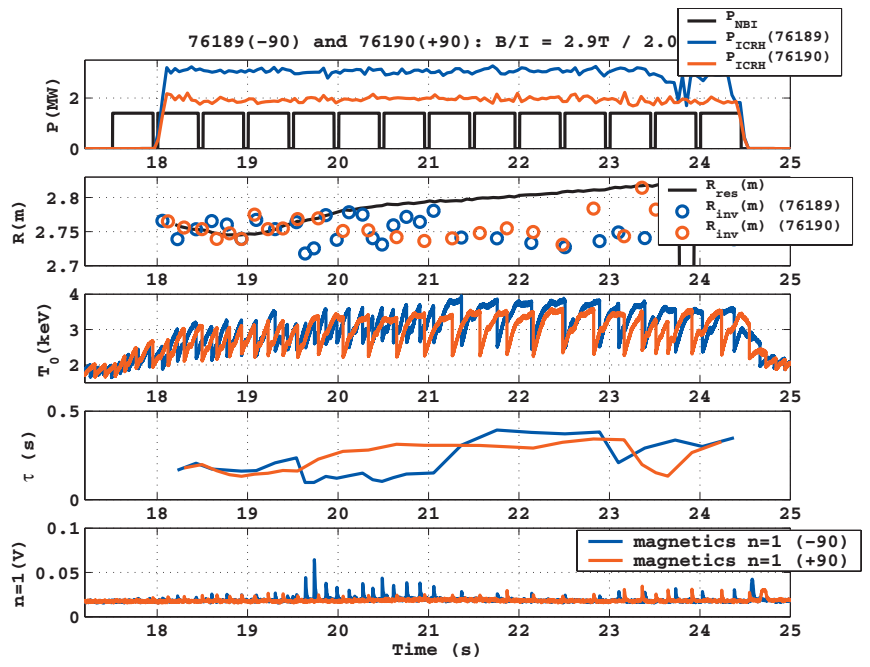

FIG. 9. (Color) Showing the time traces of NBI and ICRH power, the ${ }^{3} \mathrm{He}$ resonance position and inversion major radius, central electron temperature, sawtooth period, and $n=1$ magnetics amplitude for pulses 76189 (blue, $-90^{\circ}$ antenna phasing) and 76190 (red, $+90^{\circ}$ antenna phasing).

demonstrates sawtooth destabilization (small period) over a width of a few percent of the minor radius. For 76190 $\left(+90^{\circ}\right)$, the signature of the sawtooth stabilization is slightly broader. Nevertheless, since contrastingly different signatures occur for $+90^{\circ}$ and $-90^{\circ}$ phasings, we have demonstrated that the sawteeth were not merely modified by a change in the local conductivity, which nevertheless would not be expected to result in sawteeth that are highly sensitive to resonance position.

\section{A. Modeling of ${ }^{3} \mathrm{He}$ pulses}

Figures 10(a) and 10(b) plots the passing and trapped contributions of the fast ion current profiles for, respectively, 76189 and 76190 before the plasma drag is subtracted. It is seen that the trapped currents are very similar for co- and counterpropagating waves. This is expected, since from Eq. (25), the trapped ion current depends on the radial derivative of the distribution function, which is expected to be quite similar for the two phases (except for the pinch effect described later). However, it is seen that the radial gradient in the passing ion currents for the two phases have the opposite sign in the relevant region close to $r_{1}$. From Eq. (29) it is clear that this will lead to the opposite sign in $\delta W$ and hence the opposite effect on internal kink stability, as expected from the observed effect on the sawteeth.

The above comments are quantified in Fig. 11, where in (a) the HAGIS code calculates $\delta W$ for $76189\left(-90^{\circ}\right.$ phasing $)$ with the marker distribution taken directly from the SELFO code, and with calculations made for varying $r_{1}$ but with fixed resonance position (i.e., for varying $r_{1}-r_{\text {res }}$ as occurs in the experimental scan of $B$ ). This is compared favorably with the potential energy evaluated from Eq. (29), with the current taken from SELFO [i.e., Fig. 10(a)], and with $T_{\perp}=250 \mathrm{keV}$ and $T_{\|}=T_{\perp} / 4$. The fit of Eq. (29) recovers the trend in $\delta W$, and moreover, both are consistent with the observed variation in the sawtooth period with respect to $r_{1}-r_{\text {res }}$. Finally, Fig. 11(b) attempts to recover the trend in the sawtooth pe-

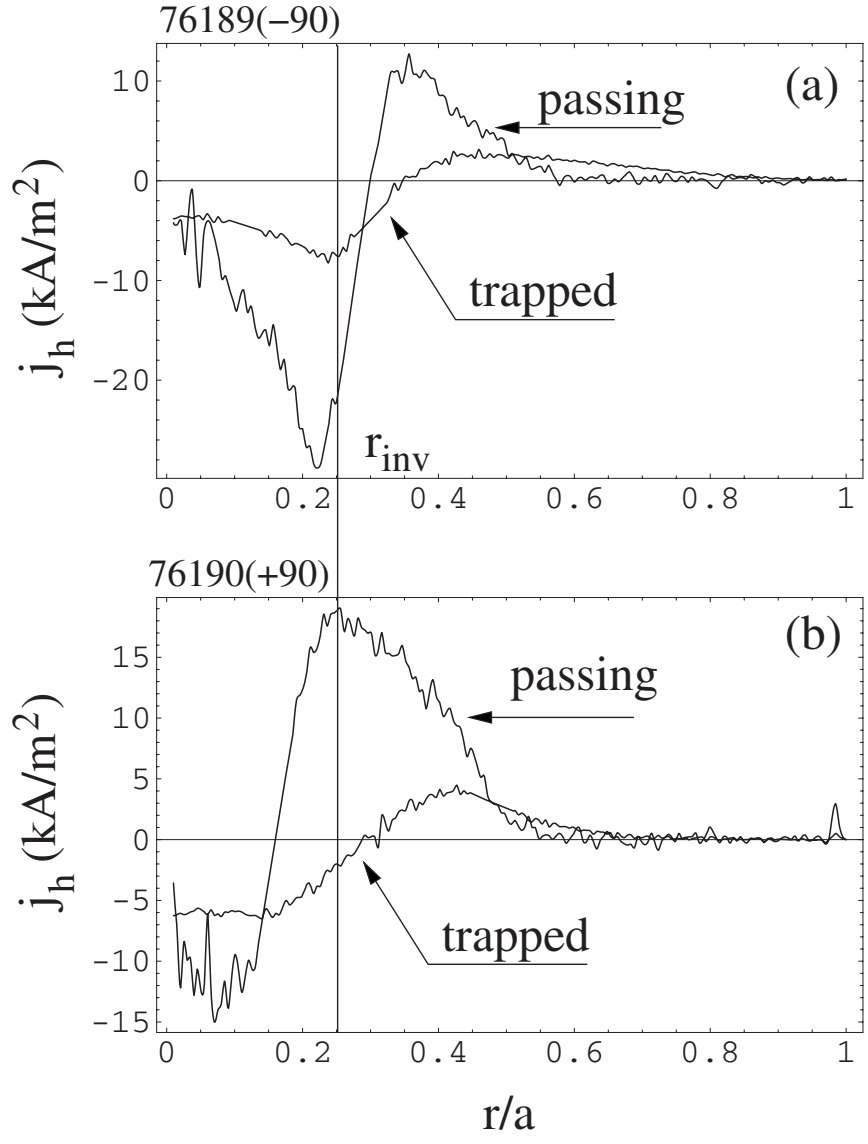

FIG. 10. Showing the passing and trapped ion currents calculated by SELFO for (a) 76189 and (b) 76190.

riod for $76190\left(+90^{\circ}\right.$ phasing). The SELFO/HAGIS simulations do this successfully. Strong internal kink stabilization occurs in a narrow region of $r_{1}$. However, the semianalytic calculation from Eq. (29) does not show such a strong variation with $r_{1}$, and this follows from the smaller negative derivative in the passing contribution of the current, shown in Fig. 10(b), in the region $r_{1}>r_{\text {res }}$. More work is needed in order to understand this discrepancy better, however, it is clear that the analysis leading to the derivation of Eq. (29) assumed a number of simplifying assumptions and models for the distribution function. Identifying the role of barely passing ions, detrapping, long tail distribution functions (non-Maxwellian tails), and nonstandard orbits will be required in that study. Nevertheless, it is pointed out here, that both the passing and trapped currents for 76190 are more on-axis than for 76189 , which is consistent with the concept of inward pinching ${ }^{37}$ of particles in the presence of copropagating waves, and it is primarily the latter that leads to the contrasting distribution functions.

\section{CONCLUSIONS}

This paper outlines in detail a new mechanism that has been proposed to explain the highly effective nature of sawtooth control using off-axis toroidally propagating ICRH. By developing an analytical treatment, initially for a general distribution function, and later with a specialized ICRH model distribution, it is shown that energetic passing ions influence 

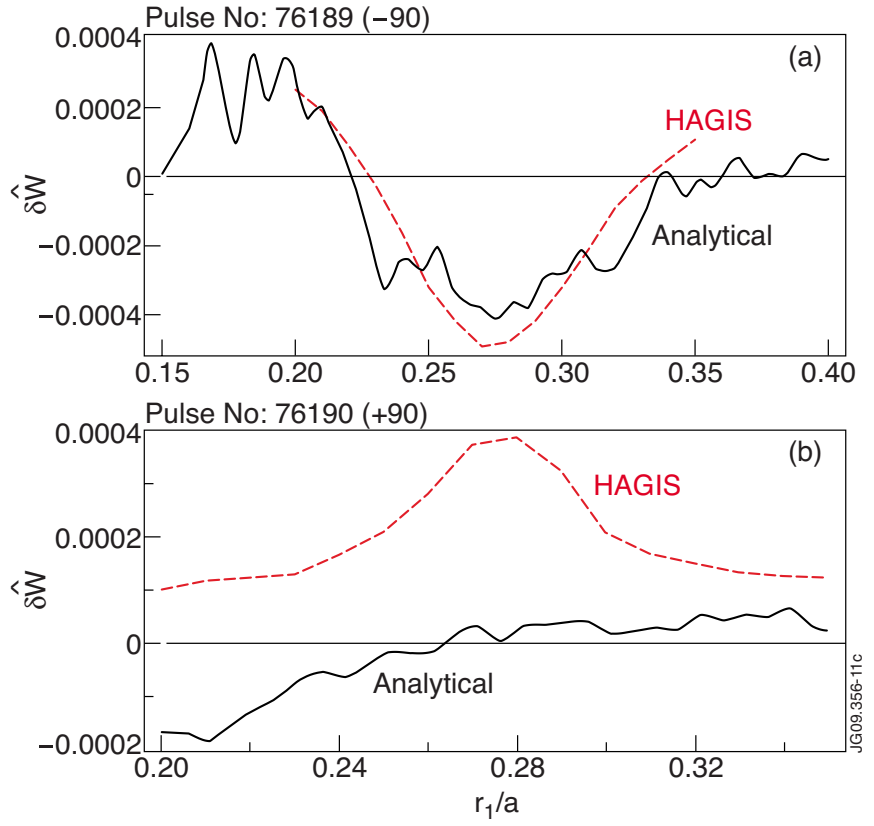

FIG. 11. (Color online) Showing the passing ion contributions to $\delta W$ for (a) 76189 and (b) 76 190. Comparisons are made between numerical solutions from the SELFO and HAGIS codes with evaluations using Eq. (29).

the internal kink mode when the distribution of ions is asymmetric in $v_{\|}$. The latter is clearly a natural feature of co- or counterpropagating ICRH waves. A JET demonstration discharge ${ }^{11}$ has been used to quantify the control mechanism, and demonstrate its viability.

In other recent discharges, ${ }^{38}$ it has been shown that a change in the magnetic field of only about $2 \%$ can be sufficient to enable or disable sawtooth control. The corresponding change in the magnetic shear has been calculated, and was shown to be quite modest, thus questioning the viability of the classical ${ }^{13}$ sawtooth control mechanism. Nevertheless, it is shown in this manuscript that when a counter propagating wave is deposited sufficiently accurately on the high field side, a newly discovered fast ion effect is so strong that the internal kink mode is driven not only resistive unstable (e.g., Ref. 2), but ideally unstable, and this in turn is consistent with measured sawteeth that are much shorter in period than those obtained in Ohmic plasmas. Furthermore, it is shown that the response of the fast ions on the internal kink mode is very sensitive to the difference between the position of the $\mathrm{RF}$ resonance position and the $q=1$ surface. This is due to the fact that the only fast ions that contribute are those that intersect the $q=1$ radius, and thus the mechanism does not rely on integrals over radial extent. Finally, the mechanism can also explain sawtooth stabilization with copropagating waves on the high field side.

It is of interest to consider the implications of this research for sawtooth control in ITER. The available range of antenna frequencies in ITER are such that ${ }^{3} \mathrm{He}$ is the likely minority species to be employed. Primarily due to the high plasma drag in plasmas with ${ }^{3} \mathrm{He}$ minority, the net current drive efficiency is expected to be poor. ${ }^{15}$ While the classical sawtooth control mechanism (e.g., Ref. 13) would fare badly from such a prediction, the fast ion mechanism outlined in this manuscript is unaffected by the bulk plasma drag current, since the orbit widths of the bulk plasma particles are negligible. ${ }^{3} \mathrm{He}$ minority ion experiments in present day machines would be a fitting test of the fast ion sawtooth control model, and the viability of ICRH control of sawteeth in ITER. Initial experiments of this sort, undertaken in JET, are presented here. It is shown that, despite weakly driven current, sawtooth control is viable with low concentration ${ }^{3} \mathrm{He}$. This work, therefore, not only serves to strengthen the theory first put forward in Ref. 9, but furthermore, gives some confidence that sawtooth control in ITER using ICRH might be viable.

\section{ACKNOWLEDGMENTS}

The authors of this work are grateful to B. Alper, M. de Baar, K. Crombe, L.-G. Eriksson, R. Felton, D. Howell, V. Kiptily, H.R. Koslowski, M.-L. Mayoral, I. Monakhov, I. Nunes, and S. D. Pinches. This work, supported by the Swiss National Science Foundation, and by the European Communities under contract of Association between EURATOM and Confederation Suisse, was carried out within the framework of the European Fusion Development Agreement. The views and opinions expressed herein do not necessarily reflect those of the European Commission.

\section{APPENDIX: ANALYTICAL EXPRESSIONS FOR POLOIDAL INTEGRALS}

The following provide closed forms for orbit averaged integrals required in the $\delta W$ expressions of this paper. $k^{2}=\left[1-\lambda B_{0}(1-\epsilon)\right] / 2 \lambda B_{0} \epsilon$ for trapped ions and $y^{2}=1 / k^{2}$ for passing ions. Now,

$$
G_{1 p}=\frac{\epsilon}{2} \oint d \theta \cos \theta(1-\epsilon \cos \theta)\left\{1-y^{2}[\sin (\theta / 2)]^{2}\right\}^{1 / 2}
$$

and

$$
G_{2 p}=\frac{y^{2}}{4} \oint d \theta \frac{\cos \theta(1-\epsilon \cos \theta)^{2}}{\left\{1-y^{2}[\sin (\theta / 2)]^{2}\right\}^{1 / 2}},
$$

where $G_{1 t}$ and $G_{2 t}$ are obtained by replacing $y^{2}$ with $1 / k^{2}$ in the corresponding equations above, and integrating over the poloidal orbit of the trapped particles. The following exact results are obtained

$$
\begin{aligned}
G_{1 t}\left(\epsilon, k^{2}\right)= & \left(\frac{2 \epsilon}{3}\right)\left[\left(1-k^{2}\right) K\left(k^{2}\right)+\left(2 k^{2}-1\right) E\left(k^{2}\right)\right] \\
& +0\left(\epsilon^{2}\right) \\
G_{1 p}\left(\epsilon, k^{2}\right)= & 2 E\left(k^{2}\right)-K\left(k^{2}\right)+\left(\frac{2 \epsilon}{3}\right) \\
& \times\left[\left(1-4 k^{2}\right) K\left(k^{2}\right)+\left(8 k^{2}-4\right) E\left(k^{2}\right)\right]+0\left(\epsilon^{2}\right),
\end{aligned}
$$




$$
\begin{aligned}
G_{1 p}\left(\epsilon, y^{2}\right)= & -\frac{2 \epsilon}{15 y^{4}}\left[\left\{5 y^{2}\left(y^{2}-2\right)+\epsilon\left(7 y^{4}+8 y^{2}-8\right)\right\} E\left(y^{2}\right)\right. \\
& \left.+\left\{2\left(y^{2}-1\right)\left[-5 y^{2}+2 \epsilon\left(y^{2}-2\right)\right]\right\} K\left(y^{2}\right)\right], \\
G_{p 2}\left(\epsilon, y^{2}\right)= & \frac{1}{15 y^{4}}\left[\left\{30 y^{4}-40 \epsilon y^{2}\left(y^{2}-2\right)+2 \epsilon^{2}\right.\right. \\
& \left.\times\left(32-32 y^{2}+17 y^{4}\right)\right\} E\left(y^{2}\right)+\left\{15 y^{4}\left(y^{2}-2\right)\right. \\
& -10 \epsilon y^{2}\left[8-8 y^{2}+3 y^{4}\right]+\epsilon^{2}\left(y^{2}-2\right) \\
& \left.\left.\times\left(32-32 y^{2}+15 y^{4}\right)\right\} K\left(y^{2}\right)\right], \\
G_{3}=\frac{\epsilon}{2} \int_{-\pi}^{\pi} & d \theta \cos \left[q(\theta)-\pi \frac{K\left[\theta / 2, y^{2}\right]}{K\left[y^{2}\right]}\right] \\
& \times(1-
\end{aligned}
$$

and

$$
\begin{aligned}
G_{4}= & \frac{y^{2}}{4} \int_{-\pi}^{\pi} d \theta \cos \left[q(\theta)-\pi \frac{K\left[\theta / 2, y^{2}\right]}{K\left[y^{2}\right]}\right] \\
& \times \frac{(1-\epsilon \cos \theta)^{2}}{\left\{1-y^{2}[\sin (\theta / 2)]^{2}\right\}^{1 / 2}} .
\end{aligned}
$$

We note that unless $y$ is very close to unity, a very good approximation is obtained by replacing $\cos [q(\theta$ $\left.\left.-\pi K\left[\theta / 2, y^{2}\right] / K\left[y^{2}\right]\right)\right]$ with unity, giving the result,

$$
\begin{aligned}
G_{3}\left(\epsilon, y^{2}\right)= & \frac{2 \epsilon}{3 y^{2}}\left[\left\{3 y^{2}+\epsilon\left(y^{2}-2\right)\right\} E\left(y^{2}\right)+\{1-\right. \\
G_{4}\left(\epsilon, y^{2}\right)= & \frac{1}{3 y^{2}}\left[\left\{-12 \epsilon y^{2}+4 \epsilon^{2}\left(y^{2}-2\right)\right\} E\left(y^{2}\right)\right. \\
& +\left\{3 y^{2}\left(4-y^{2}\right)-\epsilon\left(8 y^{2}+6 y^{4}\right)\right. \\
& \left.\left.+\epsilon^{2}\left(8+3 y^{4}\right)\right\} K\left(y^{2}\right)\right] .
\end{aligned}
$$

The trapped precession drift frequency

$$
\langle\dot{\phi}\rangle=-\frac{q \mathcal{E} F_{d}\left(r, k^{2}\right)}{r R_{0} \Omega_{c}},
$$

where

$$
\begin{aligned}
F_{d}^{t}\left(r, k^{2}\right)= & \frac{2}{1+\epsilon\left(2 k^{2}-1\right)} \\
& \times\left(H_{1}\left(k^{2}\right)+2 s(r) H_{2}\left(k^{2}\right)-\Delta^{\prime \prime}(r) H_{3}\left(k^{2}\right)-\frac{\alpha}{4 q^{2}}\right),
\end{aligned}
$$

$s=(r / q) q^{\prime}$ is the magnetic shear, $\alpha=-2 R_{0} q^{2} P^{\prime} / B_{0}^{2}$ is the ballooning parameter, while $H_{1}, H_{2}$, and $H_{3}$ are given by Eq. (10) of Ref. 23 and are,

$$
\begin{aligned}
& H_{1}=E\left(k^{2}\right) / K\left(k^{2}\right)-\frac{1}{2}, \\
& H_{2}=E\left(k^{2}\right) / K\left(k^{2}\right)+\left(k^{2}-1\right), \\
& H_{3}=\frac{2}{3}\left[\left(2 k^{2}-1\right) E\left(k^{2}\right) / K\left(k^{2}\right)+\left(1-k^{2}\right)\right] .
\end{aligned}
$$

Another result required is

$$
F_{q}=\int_{0}^{\pi / 2} d \phi \frac{\cos [2 q \arcsin (k \sin \phi)]}{\sqrt{1-k^{2} \sin ^{2} \phi}} .
$$

The following fit can be employed, ${ }^{39}$

$$
\begin{aligned}
F_{q}\left(q, k^{2}\right)= & {\left[2 E\left(k^{2}\right)-K\left(k^{2}\right)\right]-\frac{4(1-q) \cos (\pi q)}{1-4(1-q)^{2}} } \\
& \times\left[E\left(k^{2}\right)+\left(k^{2}-1\right) K\left(k^{2}\right)\right]-[1+\cos (\pi q)] f_{1}(q) \\
& \times\left[E\left(k^{2}\right)+\left(k^{2}-1\right) K\left(k^{2}\right)+\frac{2}{\pi} E\left(k^{2}\right)-1\right] \\
& -(1+\cos (\pi q))\left[E\left(k^{2}\right)-K\left(k^{2}\right)\right]-f_{2}(q)\left(1-k^{2}\right) \\
& \times\left[\frac{\pi}{2}-K\left(k^{2}\right)\right],
\end{aligned}
$$

with

$$
\begin{aligned}
& f_{1}(q)=\frac{\pi}{2}\left[1.0841-0.3193(1-q)^{2}-0.0683(1-q)^{4}\right], \\
& f_{2}(q)=5.1\left(q-\frac{1}{2}\right)(1-q)^{2}[1-0.034(1-q)] .
\end{aligned}
$$

${ }^{1}$ ITER Physics Basis Editors, ITER Physics Expert Group Chairs and CoChairs, and ITER Joint Central Team and Physics Integration Unit, Nucl. Fusion 39, 2137 (1999).

${ }^{2}$ F. Porcelli, D. Boucher, and M. N. Rosenbluth, Plasma Phys. Controlled Fusion 38, 2163 (1996).

${ }^{3}$ D. J. Campbell, D. F. H. Start, J. A. Wesson, D. V. Bartlett, V. P. Bhatnagar, M. Bures, J. G. Cordey, G. A. Cottrell, P. A. Dupperex, A. W. Edwards, C. D. Challis, C. Gormezano, C. W. Gowers, R. S. Granetz, J. H. Hammen, T. Hellsten, J. Jacquinot, E. Lazzaro, P. J. Lomas, N. Lopes Cardozo, P. Mantica, J. A. Snipes, D. Stork, P. E. Stott, P. R. Thomas, E. Thompson, K. Thomsen, and G. Tonetti, Phys. Rev. Lett. 60, 2148 (1988). ${ }^{4}$ O. Sauter, E. Westerhof, M. L. Mayoral, B. Alper, P. A. Belo, R. J. Buttery, A. Gondhalekar, T. Hellsten, T. C. Hender, D. F. Howell, T. Johnson, P. Lamalle, M. J. Mantsinen, F. Milani, M. F. F. Nave, F. Nguyen, A. L. Pecquet, S. D. Pinches, S. Podda, and J. Rapp, Phys. Rev. Lett. 88, 105001 (2002).

${ }^{5}$ F. Romanelli and R. Kamendje, on behalf of JET-EFDA Contributors, Nucl. Fusion 49, 104006 (2009); F. R. Romanelli, Proceedings of the 22nd International Conference on Fusion Energy, Geneva, 2008 [International Atomic Energy Agency (IAEA), Vienna, 2008] (all of the members of JET-EFDA Collaboration appear in the appendix of this paper).

${ }^{6}$ C. K. Phillips, J. Hosea, E. Marmar, M. W. Phillips, J. Snipesa, J. Stevens, J. Terry, J. R. Wilson, M. Bell, M. Bitter, R. Boivin, C. Bush, C. Z. Cheng, D. Darrow, E. Fredrickson, R. Goldfinger, H. Hsuan, M. Hughes, G. W. Hammett, K. Hill, D. Hoffman, W. Houlberg, H. Hsuan, M. Hughes, D. Jassby, D. McCune, K. McGuire, Y. Nagayama, D. K. Owens, H. Park, A. Ramsey, G. Schilling, J. Schivell, D. N. Smithe, B. Stratton, E. Synakowski, G. Taylor, H. Towner, R. White, and S. Zweben, Phys. Fluids B 4, 2155 (1992).

${ }^{7}$ R. B. White, P. H. Rutherford, P. Colestock, and M. N. Bussac, Phys. Rev. Lett. 60, 2038 (1988).

${ }^{8}$ J. P. Graves, Phys. Rev. Lett. 92, 185003 (2004).

${ }^{9}$ J. P. Graves, I. T. Chapman, S. Coda, L.-G. Eriksson, and T. Johnson, Phys. Rev. Lett. 102, 065005 (2009). 
${ }^{10}$ I. T. Chapman, J. P. Graves, S. D. Pinches, L. C. Appel, R. J. Hastie, T. C. Hender, S. Saarelma, S. E. Sharapov, and I. Voitsekhovitch, Phys. Plasmas 14, 070703 (2007).

${ }^{11}$ JET-EFDA Contributors, L. G. Eriksson, T. Johnson, M.-L. Mayoral, S. Coda, O. Sauter, R. J. Buttery, D. McDonald, T. Hellsten, M. J. Mantsinen, A. Mueck, J.-M. Noterdaeme, M. Santala, E. Westerhof, and P. de Vries, Nucl. Fusion, 46, S951 (2006).

${ }^{12}$ J. Hedin, T. Hellsten, L.-G. Eriksson, and T. Johnson, Nucl. Fusion 42, 527 (2002).

${ }^{13}$ V. P. Bhatnagar, D. F. H. Start, J. Jaquinot, F. Chaland, A. Cherubini, and F. Porcelli, Nucl. Fusion 34, 1579 (1994).

${ }^{14}$ E. Westerhof, O. Sauter, M. L. Mayoral, D. F. Howell, M. J. Mantsinen, M. F. F. Nave, B. Alper, C. Angioni, P. Belo, R. J. Buttery, A. Gondhalekar, T. Hellsten, T. C. Hender, T. Johnson, P. Lamalle, M. E. Maraschek, K. G. McClements, F. Nguyen, A. L. Pcquet, S. Podda, J. Rapp, S. E. Sharapov, M. Zabiego, and Contributors to EFDA JET Work Programme, Nucl. Fusion 42, 1234 (2002).

${ }^{15}$ M. Laxåback and T. Hellsten, Nucl. Fusion 45, 1510 (2005).

${ }^{16}$ N. J. Fisch, Rev. Mod. Phys. 59, 175 (1987).

${ }^{17}$ L. G. Eriksson, A. Mueck, O. Sauter, S. Coda, M. J. Mantsinen, M.-L. Mayoral, E. Westerhof, R. J. Buttery, D. McDonald, T. Johnson, J.-M. Noterdaeme, and P. de Vries, Phys. Rev. Lett. 92, 235004 (2004).

${ }^{18}$ S. D. Pinches, L. C. Appel, J. Candy, S. E. Sharapov, H. L. Berk, D. Borba, B. N. Breizman, T. C. Hender, K. I. Hopcraft, G. T. A. Huysmans, and W. Kerner, Comput. Phys. Commun. 111, 133 (1998), release version 8.08 .

${ }^{19}$ P. Helander, C. G. Gimblett, R. J. Hastie, and K. G. McClements, Phys. Plasmas 4, 2182 (1997).

${ }^{20}$ F. Porcelli, R. Stankiewicz, W. Kerner, and H. L. Berk, Phys. Plasmas 1, 470 (1994)

${ }^{21}$ R. Betti and J. P. Freidberg, Phys. Rev. Lett. 70, 3428 (1993).

${ }^{22}$ J. P. Graves, Phys. Plasmas 12, 090908 (2005).

${ }^{23}$ J. W. Connor, R. J. Hastie, and T. J. Martin, Nucl. Fusion 23, 1702 (1983).

${ }^{24}$ S. Wang, T. Ozeki, and K. Tobita, Phys. Rev. Lett. 88, 105004 (2002).

${ }^{25}$ F. S. Zaitsev, Phys. Fluids B 5, 509 (1993).

${ }^{26}$ G. D. Kerbel and M. G. McCoy, Phys. Fluids 28, 3629 (1985).

${ }^{27}$ R. Betti, Plasma Phys. Controlled Fusion 35, 941 (1993).

${ }^{28}$ I. T. Chapman, I. Jenkins, R. V. Budny, J. P. Graves, S. D. Pinches, and S. Saarelma, Plasma Phys. Controlled Fusion 50, 045006 (2008); I. T. Chapman, V. G. Igochine, J. P. Graves, S. D. Pinches, A. Gude, I. Jenkins, M.
Maraschek, G. Tardini, and ASDEX Upgrade Team, JET EFDA Contributors, Nucl. Fusion 49, 035006 (2009).

${ }^{29}$ G. J. Kramer, M. Iwase, Y. Kusama, A. Morioka, M. Nemoto, T. Nishitani, K. Shinohara, S. Takeji, K. Tobita, T. Ozeki, C. Z. Cheng, G.-Y. Fu, and R. Nazikian, Nucl. Fusion 40, 1383 (2000).

${ }^{30}$ J. P. Graves, C. Angioni, R. V. Budny, R. J. Buttery, S. Coda, L.-G. Eriksson, C. G. Gimblett, T. P. Goodman, R. J. Hastie, M. A. Henderson, H. R. Koslowski, M. J. Mantsinen, A. Martynov, M.-L. Mayoral, A. Muck, M. F. F. Nave, O. Sauter, E. Westerhof, and JETEFDA Contributors, Plasma Phys. Controlled Fusion 47 B121 (2005).

${ }^{31}$ J. P. Graves, K. I. Hopcraft, R. O. Dendy, R. J. Hastie, K. G. McClements, and M. Mantsinen, Phys. Rev. Lett. 84, 1204 (2000).

${ }^{32}$ W. A. Cooper, J. P. Graves, S. P. Hirshman, T. Yamaguchi, Y. Narushima, S. Okamura, S. Sakakibara, C. Suzuki, K. Y. Watanabe, H. Yamada, and K. Yamazaki, Nucl. Fusion 46, 683 (2006).

${ }^{33}$ W. A. Cooper, J. P. Graves, T. M. Tran, R. Gruber, T. Yamaguchi, Y. Narushima, S. Okamura, S. Sakakibara, C. Suzuki, K. Y. Watanabe, H. Yamada, and K. Yamazaki, Fusion Sci. Technol. 50, 245 (2006).

${ }^{34}$ T. Hellsten, J. Carlsson, and L.-G. Eriksson, Phys. Rev. Lett. 74, 3612 (1995).

${ }^{35}$ A. Nocentini, M. Tessarotto, and F. Engelmann, Nucl. Fusion 15, 359 (1975).

${ }^{36}$ J. P. Graves, I. T. Chapman, S. Coda, T. Johnson, M. Lennholm, B. Alper, M. de Baar, K. Crombe, L.-G. Eriksson, R. Felton, D. Howell, V. Kiptily, H. R. Koslowski, M.-L. Mayoral, I. Monakhov, I. Nunes, and S. D. Pinches, Nucl. Fusion 50, 052002 (2010).

${ }^{37}$ L.-G. Eriksson, M. Mantsinen, D. Borba, A. Fasoli, R. Heeter, S. Sharapov, D. F. H. Start, J. Carlsson, A. Gondhalekar, T. Hellsten, and A. Korotkov, Phys. Rev. Lett. 81, 1231 (1998).

${ }^{38}$ S. Coda, L.-G. Eriksson, M. Lennholm, J. P. Graves, T. Johnson, J. H. Brzozowski, M. DeBaar, D. F. Howell, S. Jachmich, V. Kiptily, R. Koslowski, M.-L. Mayoral, A. Mueck, S. Pinches, G. Saibene, M. I. K. Santala, M. F. Stamo, M. Valisa, and JET-EFDA Contributors, Proceedings of Contributed Papers, 34th European Physical Society Plasma Physics Conference, Warsaw, 2007, edited by P. Gasior and J. Wolowski (unpublished); Proceedings of the International Conference on Plasma Physics, Omsk, 1986, edited by I. I. Ivanovich (unpublished), Vol. 31F, p. 5.130.

${ }^{39}$ J. P. Graves, R. J. Hastie, and K. I. Hopcraft, Plasma Phys. Controlled Fusion 42, 1049 (2000). 